\title{
Constraints on $f(R)$ theory and Galileons from the latest data of galaxy redshift surveys
}

\author{
Hiroyuki Okada, ${ }^{1}$ Tomonori Totani, ${ }^{1,2}$ and Shinji Tsujikawa ${ }^{3}$ \\ ${ }^{1}$ Department of Astronomy, Kyoto University, Kitashirakawa-Oiwake-cho, Sakyo-ku, Kyoto 606-8502, Japan \\ ${ }^{2}$ Department of Astronomy, The University of Tokyo, Hongo, Bunkyo-ku, Tokyo 113-0033, Japan \\ ${ }^{3}$ Department of Physics, Faculty of Science, Tokyo University of Science, \\ 1-3, Kagurazaka, Shinjuku-ku, Tokyo 162-8601, Japan
}

(Dated: August 6, 2018)

\begin{abstract}
The growth rate of matter density perturbations has been measured from redshift-space distortion (RSD) in the galaxy power spectrum. We constrain the model parameter space for representative modified gravity models to explain the dark energy problem by using the recent data of $f_{m}(z) \sigma_{8}(z)$ at the redshifts $z=0.06-0.8$ measured by WiggleZ, SDSS LRG, BOSS, and 6dFGRS. We first test the Hu-Sawicki's $f(R)$ dark energy model, and find that only the parameter region close to the standard $\Lambda$ Cold Dark Matter $(\Lambda \mathrm{CDM})$ model is allowed $(\lambda>12$ and 5 for $n=1.5$ and 2 , respectively, at $95 \%$ $\mathrm{CL}$ ). We then investigate the covariant Galileon model with a de Sitter attractor and show that the parameter space consistent with the background expansion history is excluded by the RSD data at more than $8 \sigma$ because of the too large growth rate predicted by the theory. Finally, we consider the extended Galileon scenario, and we find that, in contrast to the covariant Galileon, there is a model parameter space for a tracker solution that is consistent with the RSD data within a $2 \sigma$ level.
\end{abstract}

\section{INTRODUCTION}

The observational support for the existence of dark energy [1 3] has motivated the idea that the gravitational law may be modified from General Relativity (GR) at large distances to realize the late-time cosmic acceleration (see Refs. [4 7] for reviews). In this vein many modified gravity models of dark energy have been proposed-including those based on $f(R)$ gravity [8], scalar-tensor theories [9], the Dvali-Gabadadze-Porrati (DGP) braneworld scenario [10], Galileons [11, 12], and so on.

Measuring the growth rate of large scale structures in the Universe gives a strong test for the modified gravity scenario, because modified gravity models generally predict different growth rates from that in the standard $\Lambda$ Cold Dark Matter $(\Lambda \mathrm{CDM})$ model. In fact the equations of linear matter density perturbations have been derived for a number of modified gravity models - including $f(R)$ gravity [13, 14], the DGP model [15], and Galileons [16. Recently De Felice et al. 17] derived the full perturbation equations as well as the effective gravitational coupling to non-relativistic matter in the most general scalar-tensor theories in 4 dimensions [18 20] (which cover most of the single-field dark energy models proposed in the literature).

One of the methods to measure the cosmic growth rate is redshift-space distortion (RSD) that appears in clustering pattern of galaxies in galaxy redshift surveys because of radial peculiar velocities. RSD on large and linear scales reflects the velocity of inward collapse motion of large scale structure, which is directly related to the evolutionary speed of matter overdensity perturbations simply from the mass conservation [21]. Recent galaxy redshift surveys have provided measurements of the growth rate $f_{m}(z)$ or $f_{m}(z) \sigma_{8}(z)$ as a function of redshift up to $z \sim 122[33]$, where $f_{m}=d \ln \delta_{m} / d \ln a$, $\delta_{m}$ is the fractional over-density of non-relativistic matter, $a$ is the scale factor of the Universe, and $\sigma_{8}$ is the rms amplitude of over-density at the comoving $8 h^{-1}$ Mpc scale ( $h$ is the normalized Hubble parameter $H_{0}=$ $\left.100 h \mathrm{~km} \mathrm{sec}^{-1} \mathrm{Mpc}^{-1}\right)$. In this paper, we constrain some of the modified gravity models proposed to solve the dark energy problem, by using the latest RSD data of 2 dFGRS [23], WiggleZ [27], SDSS LRG [28], BOSS [29], and 6dFGRS [30].

In this paper, we choose the $f(R)$ gravity and Galileon models as the representative theories of modified gravity. A general difficulty of dark energy models based on modified gravity is the emergence of "the fifth force" that can violate the constraints from local gravity experiments. There are a number of mechanisms to suppress the propagation of the fifth force in local regions: (i) chameleon mechanism [34], (ii) Vainshtein mechanism 35], and (iii) the symmetron mechanism 36, 37]. The symmetron mechanism is irrelevant to dark energy because the energy scale of its simplest potential is too small to be used for the late-time cosmic acceleration ${ }^{1}$. The $f(R)$ gravity and Galileons are representative models to explain the acceleration, avoiding the fifth force problem by mechanisms (i) and (ii), respectively (see Sec. II, where we give a brief review of these theories).

In $f(R)$ gravity several authors put observational bounds on the parameter $B=\left(F_{R} \dot{R} / F\right)(H / \dot{H})$ by assuming the $\Lambda \mathrm{CDM}$ background 39 41], where $F=$ $\partial f / \partial R, F_{, R}=\partial F / \partial R$, and $H$ is the Hubble parameter (see also Refs. [42] for related works). At the level of perturbations the parameter $B$ characterizes the de-

\footnotetext{
${ }^{1}$ Unless the field potential is carefully designed, this problem even persists for the chameleon field 38].
} 
viation from the $\Lambda \mathrm{CDM}$ model. The joint data analysis of cluster abundance, the cosmic microwave background (CMB), and other observations shows that the value of $B$ today is constrained to be $B_{0}<1.1 \times 10^{-3}$ at the $95 \%$ confidence level (CL) [41]. The matter and the velocity power spectra were also computed with $N$-body simulations for the Hu-Sawicki model (a popular and viable $f(R)$ dark energy model, see Eq. (6) below) with $n=1 / 2$ [43]. However, those past works did not place explicit constraints on the viable parameter space of the Hu-Sawicki model from the RSD data.

Recently the covariant Galileon dark energy model (whose cosmological dynamics was studied in Refs. 44 [46]) was confronted with observations [47] by using the RSD data of WiggleZ and BOSS as well as the data of Supernovae Ia (SN Ia), CMB, and Baryon Acoustic Oscillations (BAO). It was found that this model is severely disfavored over the $\Lambda \mathrm{CDM}$. In this paper we show that the covariant Galileon is indeed excluded at more than $8 \sigma$ CL by using the most recent RSD data of SDSS LRG and 6dFGRS in addition to the WiggleZ and BOSS data. We then further consider the extended Galileon scenario in which a tracker solution with an arbitrary constant dark energy equation of state smaller than -1 is realized during the matter era. Unlike the covariant Galileon we show that there are some viable parameter spaces compatible with the current observational data of RSD as well as SN Ia, CMB, and BAO.

This paper is organized as follows. In Sec. II we review the basic properties of dark energy models based on $f(R)$ gravity and Galileons. In Sec. III we study the evolution of the growth rate of matter perturbations in these models. In Sec. IV we put observational constraints on the parameters of each model, and Sec. $\mathrm{V}$ is devoted to conclusions.

\section{MODIFIED GRAVITY MODELS}

In general the modified gravity models of dark energy are required to recover the Newton gravity at short distances for the consistency with local gravity experiments in the solar system [48]. As we mentioned in Introduction, there are two known mechanisms for the recovery of the Newton gravity in local regions.

One is the chameleon mechanism [34], under which the mass of a scalar degree of freedom is different depending on the matter densities in the surrounding environment. If the effective mass is sufficiently large in the regions of high density, the coupling between the field and nonrelativistic matter can be suppressed by having a thinshell inside a spherically symmetric body.

In $f(R)$ gravity there exists a scalar degree of freedom ("scalaron" [49]) with a potential of gravitational origin for nonlinear functions $f$ in terms of the Ricci scalar $R$. In this case it is possible to design the scalar potential such that the chameleon mechanism works at short distances by choosing appropriate forms of $f(R)[38,50,51]$.
Explicit $f(R)$ models of dark energy that can satisfy both local gravity and cosmological constraints have been proposed in Refs. [52 [56].

Another mechanism for the recovery of Newton gravity is the Vainshtein mechanism [35], under which nonlinear scalar-field self interactions can suppress the propagation of the fifth force at short distances even in the absence of the field potential. In the DGP model, a brane-bending mode $\phi$ gives rise to the self-interaction of the form $X \square \phi$ (where $\left.X=-(\nabla \phi)^{2} / 2\right)$ through the mixture with a transverse graviton [57]. This self-interaction leads to the decoupling of the field $\phi$ from matter within a radius much larger than the solar-system scale [58]. However this model is plagued by the ghost problem [59], in addition to the incompatibility with cosmological constraints at the background level [60].

The field self-interaction $X \square \phi$, which is crucial for the recovery of GR in local regions, gives rise to the field equations invariant under the Galilean shift $\phi(x) \rightarrow$ $\phi(x)+b_{\mu} x^{\mu}+c$ in flat spacetime. Nicolis et al. [11] derived the general field Lagrangian by imposing the Galilean symmetry in Minkowski spacetime. Deffayet et al. 12] obtained the covariant version of the Galileon Lagrangian keeping the field equations up to second order in curved backgrounds (see Eq. (11) below). In this case the Galilean symmetry is recovered in the limit of Minkowski spacetime. For the covariant Galileon the presence of field self interactions other than $X \square \phi$ gives rise to the theoretically allowed parameter space in which ghosts and instabilities are absent [45, 46]. There exists the allowed parameter region in which the background cosmological constraints are satisfied [61].

If we extend the Galileon self-interaction $X \square \phi$ to the form $X^{p} \square \phi$ [62], it gives rise to a scenario equivalent to the Dvali-Turner model at the background level [63]. In this case the dark energy equation of state is $w_{\mathrm{DE}}=-1-1 /(2 p-1)$ during the matter era. However there is an anti-correlation between the large-scale structure (LSS) and the Integrated-Sachs-Wolfe (ISW) effect in $\mathrm{CMB}$, which provides a tight bound on $p$, as $p>4.2 \times 10^{3}(95 \% \mathrm{CL})$ [64], in which case the model is practically indistinguishable from the $\Lambda \mathrm{CDM}$. This situation is alleviated by taking into account other extended Galileon terms [65]. In this extended Galileon scenario there exist viable parameter spaces in which the LSS and ISW are positively correlated [66], so that $w_{\mathrm{DE}}$ does not need to be extremely close to -1 .

In what follows we review the dark energy models based on $f(R)$ gravity, covariant Galileon, and extended Galileon, respectively.

\section{A. $f(R)$ gravity}

The first class is $f(R)$ gravity [49, 67] given by the action

$$
S=\frac{M_{\mathrm{pl}}^{2}}{2} \int d^{4} x \sqrt{-g} f(R)+S_{m},
$$


where $M_{\mathrm{pl}}$ is the reduced Planck mass, $g$ is a determinant of the spacetime metric $g_{\mu \nu}, f(R)$ is a function of the Ricci scalar $R$, and $S_{m}$ is the action of non-relativistic matter. We assume that non-relativistic matter (with a negligible pressure) is described by a barotropic perfect fluid minimally coupled to gravity. We focus on the metric formalism under which the action (11) is varied with respect to $g_{\mu \nu}$ (see Refs. [4, 5] for reviews).

On the flat Friedmann-Lemaitre-Robertson-Walker (FLRW) background with the line-element $d s^{2}=-d t^{2}+$ $a^{2}(t) d \boldsymbol{x}^{2}$ the field equations following from the action (11) are [5]

$$
\begin{aligned}
3 F H^{2} & =(F R-f) / 2-3 H \dot{F}+\rho_{m} / M_{\mathrm{pl}}^{2}, \\
-2 F \dot{H} & =\ddot{F}-H \dot{F}+\rho_{m} / M_{\mathrm{pl}}^{2},
\end{aligned}
$$

where $F=\partial f / \partial R, H=\dot{a} / a$ is the Hubble parameter, $\rho_{m}$ is the energy density of non-relativistic matter, $R=6\left(2 H^{2}+\dot{H}\right)$, and a dot represents a derivative with respect to cosmic time $t$. From these equations we can also obtain the continuity equation $\dot{\rho}_{m}+$ $3 H \rho_{m}=0$. Defining the density parameters $\Omega_{\mathrm{DE}}=$ $(F R-f) /\left(6 F H^{2}\right)-\dot{F} /(H F)$ and $\Omega_{m}=\rho_{m} /\left(3 F H^{2} M_{\mathrm{pl}}^{2}\right)$, it follows that $\Omega_{\mathrm{DE}}+\Omega_{m}=1$ from Eq. (2).

When we apply $f(R)$ gravity to dark energy the model is usually constructed to possess de Sitter solutions characterized by $R=R_{\mathrm{dS}}=12 H_{\mathrm{dS}}^{2}=$ constant, where $H_{\mathrm{dS}}$ is the Hubble parameter at the de Sitter solution. Using Eq. (2) with $\rho_{m}=0$, it follows that

$$
R_{\mathrm{dS}} F\left(R_{\mathrm{dS}}\right)=2 f\left(R_{\mathrm{dS}}\right)
$$

where $R_{\mathrm{dS}}$ is the similar order to the Hubble parameter $H_{0}$ today. Considering the homogeneous perturbations around $R=R_{\mathrm{dS}}$, the de Sitter solution is stable under the following condition $68-70]$ :

$$
0<R F_{, R}(R) / F(R) \leq 1,
$$

in which case the solutions with different initial conditions converge to the de Sitter attractor.

For the construction of viable $f(R)$ dark energy models we require the condition $F(R)>0$ to avoid the appearance of ghosts [5]. The mass squared of a scalar degree of freedom is given by $M^{2} \simeq\left(3 F_{, R}(R)\right)^{-1}$ in the regime $M^{2} \gg R$, so that the condition $F_{, R}(R)>0$ needs to be satisfied to be free from tachyonic instabilities [53]. In the regions of high density $\left(\rho \gg H_{0}^{2} M_{\mathrm{pl}}^{2}\right)$ the mass $M$ needs to be much larger than $H_{0}$, so that the chameleon mechanism can be at work to suppress the propagation of the fifth force.

A typical example satisfying the above demands is given by [52]

$$
f(R)=R-\lambda R_{c} \frac{\left(R / R_{c}\right)^{2 n}}{\left(R / R_{c}\right)^{2 n}+1},
$$

where $n, \lambda, R_{c}$ are positive constants. There exist other models such as $f(R)=R-\lambda R_{c}\left[1-\left(1+R^{2} / R_{c}^{2}\right)^{-n}\right]$ $(n>0)$ [53] and $f(R)=R-\lambda R_{c} \tanh \left(R / R_{c}\right)$ [55], but the basic properties are similar to those in the model (6) (the latter corresponds to the limit $n \gg 1$ ). The model $f(R)=R-\lambda R_{c}\left(R / R_{c}\right)^{p}(0<p<1)[70$, 71] is also cosmologically viable, but local gravity constraints put the severe bound $p<10^{-10}$ [51] (i.e., indistinguishable from the $\Lambda \mathrm{CDM}$ model). For the model (6) the modification of gravity manifests itself even for $n$ of the order of unity.

In the following we focus on the model (6). The condition (4) for the existence of de Sitter solutions is given by

$$
\lambda=\frac{\left(1+\mathcal{R}_{\mathrm{dS}}^{2 n}\right)^{2}}{\mathcal{R}_{\mathrm{dS}}^{2 n-1}\left(2+2 \mathcal{R}_{\mathrm{dS}}^{2 n}-2 n\right)},
$$

where $\mathcal{R}_{\mathrm{dS}}=R_{\mathrm{dS}} / R_{c}$. The stability condition (5) reads

$$
2 \mathcal{R}_{\mathrm{dS}}^{4 n}-(2 n-1)(2 n+4) \mathcal{R}_{\mathrm{dS}}^{2 n}+(2 n-1)(2 n-2) \geq 0 .
$$

For given $n$ this gives the lower bounds on $\mathcal{R}_{\mathrm{dS}}$ and $\lambda$. If $n=1$, for example, one has $\mathcal{R}_{\mathrm{dS}} \geq \sqrt{3}$ and $\lambda \geq 8 \sqrt{3} / 9$. For $\lambda$ and $n$ of the order of unity, Eq. (17) shows that $R_{\mathrm{dS}}$ is roughly of the order of $R_{c}$. In the limit that $R \gg R_{c}$ the model (6) behaves as $f(R) \simeq R-\lambda R_{c}\left[1+\left(R / R_{c}\right)^{-2 n}\right]$, which is close to the $\Lambda$ CDM model.

In the regions of high density the chameleon mechanism works for the model (6), in which case the fifth force was evaluated in Refs. [51, 52]. From the local gravity constraints using the violation of weak equivalence principle, we obtain the following bound [51]:

$$
n>0.9 \text {. }
$$

Note that $n$ and $\lambda$ are the two fundamental parameters for the model (6). For those parameters the ratio $\mathcal{R}_{\mathrm{dS}}=R_{\mathrm{dS}} / R_{c}$ is known from Eq. (7), which leads to the estimation $R_{c} \approx H_{0}^{2} / \mathcal{R}_{\mathrm{dS}}$. Theoretically natural model parameters are $n$ and $\lambda$ of the order of unity, but it remains to see whether such parameter space can be consistent with the RSD data.

\section{B. Covariant Galileon}

The second class of dark energy models is the covariant Galileon [11, 12]. This theory is the subclass of the Horndeski's general action [18 20] characterized by

$$
S=\sum_{i=2}^{5} \int d^{4} x \sqrt{-g} \mathcal{L}_{i}+S_{m}
$$

where

$$
\begin{aligned}
\mathcal{L}_{2}= & K(\phi, X), \quad \mathcal{L}_{3}=-G_{3}(\phi, X) \square \phi, \\
\mathcal{L}_{4}= & G_{4}(\phi, X) R+G_{4, X}\left[(\square \phi)^{2}-\left(\nabla_{\mu} \nabla_{\nu} \phi\right)\left(\nabla^{\mu} \nabla^{\nu} \phi\right)\right], \\
\mathcal{L}_{5}= & G_{5}(\phi, X) G_{\mu \nu}\left(\nabla^{\mu} \nabla^{\nu} \phi\right) \\
& -\left(G_{5, X} / 6\right)\left[(\square \phi)^{3}-3(\square \phi)\left(\nabla_{\mu} \nabla_{\nu} \phi\right)\left(\nabla^{\mu} \nabla^{\nu} \phi\right)\right. \\
& \left.+2\left(\nabla^{\mu} \nabla_{\alpha} \phi\right)\left(\nabla^{\alpha} \nabla_{\beta} \phi\right)\left(\nabla^{\beta} \nabla_{\mu} \phi\right)\right] .
\end{aligned}
$$


Here $K$ and $G_{i}(i=3,4,5)$ are functions in terms of a scalar field $\phi$ and its kinetic energy $X=-g^{\mu \nu} \partial_{\mu} \phi \partial_{\nu} \phi / 2$, and $G_{\mu \nu}$ is the Einstein tensor. The covariant Galileon [12] corresponds to the choice

$$
\begin{aligned}
& K=c_{1} M^{3} \phi-c_{2} X, \\
& G_{3}=c_{3} X / M^{3}, \\
& G_{4}=M_{\mathrm{pl}}^{2} / 2-c_{4} X^{2} / M^{6}, \\
& G_{5}=3 c_{5} X^{2} / M^{9},
\end{aligned}
$$

where $c_{i}$ 's are dimensionless constants, and $M$ is a constant having a dimension of mass ${ }^{2}$. Since we are interested in the case where the cosmic acceleration is driven by field kinetic terms without a potential, we set $c_{1}=0$ in the following discussion. Note that the Einstein-Hilbert term $M_{\mathrm{pl}}^{2} R / 2$ appears inside the Lagrangian $\mathcal{L}_{4}$.

On the flat FLRW background the equations of motion for the covariant Galileon are 45

$$
\begin{aligned}
& 3 M_{\mathrm{pl}}^{2} H^{2}=\rho_{\mathrm{DE}}+\rho_{m}, \\
& 3 M_{\mathrm{pl}}^{2} H^{2}+2 M_{\mathrm{pl}}^{2} \dot{H}=-P_{\mathrm{DE}},
\end{aligned}
$$

where

$$
\begin{aligned}
\rho_{\mathrm{DE}} \equiv & -c_{2} \dot{\phi}^{2} / 2+3 c_{3} H \dot{\phi}^{3} / M^{3}-45 c_{4} H^{2} \dot{\phi}^{4} /\left(2 M^{6}\right) \\
& +21 c_{5} H^{3} \dot{\phi}^{5} / M^{9} \\
P_{\mathrm{DE}} \equiv & -c_{2} \dot{\phi}^{2} / 2-c_{3} \dot{\phi}^{2} \ddot{\phi} / M^{3} \\
& +3 c_{4} \dot{\phi}^{3}\left[8 H \ddot{\phi}+\left(3 H^{2}+2 \dot{H}\right) \dot{\phi}\right] /\left(2 M^{6}\right) \\
& -3 c_{5} H \dot{\phi}^{4}\left[5 H \ddot{\phi}+2\left(H^{2}+\dot{H}\right) \dot{\phi}\right] / M^{9}
\end{aligned}
$$

The dark energy equation of state $w_{\mathrm{DE}}$ is defined as $w_{\mathrm{DE}}=P_{\mathrm{DE}} / \rho_{\mathrm{DE}}$.

The de Sitter solution $\left(H=H_{\mathrm{dS}}=\right.$ constant $)$ is realized for $\dot{\phi}=\dot{\phi}_{\mathrm{dS}}=$ constant. Normalizing the mass $M$ to be $M^{3}=M_{\mathrm{pl}} H_{\mathrm{dS}}^{2}$ and defining $x_{\mathrm{dS}} \equiv \dot{\phi}_{\mathrm{dS}} /\left(H_{\mathrm{dS}} M_{\mathrm{pl}}\right)$, Eqs. (16) and (17) lead to the following relations at the de Sitter solution [45]

$$
\begin{aligned}
& c_{2} x_{\mathrm{dS}}^{2}=3(2+3 \alpha-4 \beta), \\
& c_{3} x_{\mathrm{dS}}^{3}=2+9(\alpha-\beta),
\end{aligned}
$$

where

$$
\alpha \equiv c_{4} x_{\mathrm{dS}}^{4}, \quad \beta \equiv c_{5} x_{\mathrm{dS}}^{5} .
$$

The relations (20) and (21) are not subject to change under the rescaling $x_{\mathrm{dS}} \rightarrow \gamma x_{\mathrm{dS}}, \quad c_{2} \rightarrow c_{2} / \gamma^{2}$, and $c_{3} \rightarrow c_{3} / \gamma^{3}$, where $\gamma$ is a real number. Hence, the rescaled choices of $c_{2}$ and $c_{3}$ lead to the same cosmological dynamics. This means that we can set $x_{\mathrm{dS}}=1$ without loss of generality, in which case $c_{2}$ and $c_{3}$ are

\footnotetext{
${ }^{2}$ In the original paper of Galileons [1] the linear potential $c_{1} M^{3} \phi$ is expressed as a separate Lagrangian $\mathcal{L}_{1}$. Here we include both the potential and the kinetic term inside $\mathcal{L}_{2}$.
}

directly known from Eqs. (20) and (21). It is convenient to use the two model parameters $\alpha$ and $\beta$ because the coefficients of physical quantities can be expressed in terms of them.

We also note that the de Sitter solution given above is always stable against homogenous perturbations [45, 46], so the solutions with different initial conditions finally converge to the de Sitter attractor. If we do not demand the existence of de Sitter solutions, there are more parameter spaces left for the coefficients $c_{2}$ and $c_{3}$. In such cases, however, the existence of a stable accelerated attractor is not generally guaranteed.

Introducing the two variables $r_{1}=\dot{\phi}_{\mathrm{dS}} H_{\mathrm{dS}} /(\dot{\phi} H)$ and $r_{2}=\left(\dot{\phi} / \dot{\phi}_{\mathrm{dS}}\right)^{4} / r_{1}$, which satisfy $r_{1}=r_{2}=1$ at the de Sitter solution, the dark energy density parameter $\Omega_{\mathrm{DE}}=$ $\rho_{\mathrm{DE}} /\left(3 M_{\mathrm{pl}}^{2} H^{2}\right)$ can be expressed as

$$
\begin{aligned}
\Omega_{\mathrm{DE}}= & -(2+3 \alpha-4 \beta) r_{1}^{3} r_{2} / 2+(2+9 \alpha-9 \beta) r_{1}^{2} r_{2} \\
& -15 \alpha r_{1} r_{2} / 2+7 \beta r_{2} .
\end{aligned}
$$

The autonomous equations for the variables $r_{1}$ and $r_{2}$ were derived in Ref. [46]. There are three distinct fixed points for this dynamical system: (A) $\left(r_{1}, r_{2}\right)=(0,0)$, (B) $\left(r_{1}, r_{2}\right)=(1,0)$, and (C) $\left(r_{1}, r_{2}\right)=(1,1)$. Point $(\mathrm{C})$ is the de Sitter solution, which is always stable against homogeneous perturbations. Point (B) corresponds to a tracker solution characterized by $\dot{\phi} \propto 1 / H$. For the initial conditions close to the fixed point (A) the solutions converge to the tracker once $r_{1}$ approaches 1 . The fixed point (B) is followed by the de Sitter point (C) after $r_{2}$ grows to the order of 1 . The epoch at which the solutions approach the tracker depends on the initial values of $r_{1}$. For smaller $r_{1}$ the tracking occurs later.

Along the tracker one has $\Omega_{\mathrm{DE}}=r_{2}$ and $w_{\mathrm{DE}}=$ $-2 /\left(1+\Omega_{\mathrm{DE}}\right)$ after the radiation-dominated epoch. In the regime close to the fixed point (A) the dark energy equation of state is given by $w_{\mathrm{DE}}=-1 / 8$ during the matter era. After the solutions reach the tracker, $w_{\mathrm{DE}}$ changes from -2 (matter era) to -1 (de Sitter era).

The joint data analysis of SN Ia, CMB, and BAO shows that the tracker is disfavored from the observational data of the distance measurement 61. For the compatibility with the data the solutions need to approach the tracker at late times with the minimum value of $w_{\mathrm{DE}}$ larger than -1.3 . Taking into account the conditions for the avoidance of ghosts and Laplacian instabilities [45, 46], the parameters $\alpha$ and $\beta$ are constrained to be

$$
\alpha=1.404 \pm 0.057, \quad \beta=0.419 \pm 0.023,
$$

from the joint data analysis of SN Ia (Union2), CMB, and BAO 61].

We stress that, for given initial conditions of $r_{1}$ and $r_{2}$ (in other words, given initial conditions of $\dot{\phi}$ and $H$ ) under which the solutions enter the tracker at late times, there are two parameters $\alpha$ and $\beta$ left for the likelihood analysis in Sec. IV] The bounds (24) will be used for such an analysis. 


\section{Extended Galileon}

The extended Galileon [65] is described by the Horndeski's Lagrangian (11) with the choice

$$
\begin{aligned}
& K=-c_{2} M_{2}^{4\left(1-p_{2}\right)} X^{p_{2}}, \\
& G_{3}=c_{3} M_{3}^{1-4 p_{3}} X^{p_{3}}, \\
& G_{4}=M_{\mathrm{pl}}^{2} / 2-c_{4} M_{4}^{2\left(1-2 p_{4}\right)} X^{p_{4}}, \\
& G_{5}=3 c_{5} M_{5}^{-\left(1+4 p_{5}\right)} X^{p_{5}},
\end{aligned}
$$

where $c_{i}$ and $p_{i}(i=2, \cdots, 5)$ are dimensionless constants, and $M_{i}$ 's are constants having a dimension of mass. The covariant Galileon corresponds to $p_{2}=p_{3}=1$ and $p_{4}=p_{5}=2$. In Ref. [65] it was shown that there exists a tracker solution with $H \dot{\phi}^{2 q}=$ constant $(q>0)$ for the powers

$$
\begin{array}{ll}
p_{2}=p, & p_{3}=p+(2 q-1) / 2, \\
p_{4}=p+2 q, & p_{5}=p+(6 q-1) / 2 .
\end{array}
$$

Note that the tracker corresponds to an attractor where the solutions with different initial conditions converge to a common trajectory ( $p=1$ and $q=1 / 2$ for the covariant Galileon).

The extended Galileon possesses de Sitter solutions with $H=H_{\mathrm{dS}}=$ constant and $\dot{\phi}=\dot{\phi}_{\mathrm{dS}}=$ constant. We relate the masses $M_{i}$ 's in Eqs. (25)- 28) with $H_{\mathrm{dS}}$, as $M_{2}=\left(H_{\mathrm{dS}} M_{\mathrm{pl}}\right)^{1 / 2}, M_{3}=\left(H_{\mathrm{dS}}^{-2 p_{3}} M_{\mathrm{pl}}^{1-2 p_{3}}\right)^{1 /\left(1-4 p_{3}\right)}$, $M_{4}=\left(H_{\mathrm{dS}}^{-2 p_{4}} M_{\mathrm{pl}}^{2-2 p_{4}}\right)^{1 /\left(2-4 p_{4}\right)}$, and $M_{5}=$ $\left(H_{\mathrm{dS}}^{2+2 p_{5}} M_{\mathrm{pl}}^{2 p_{5}-1}\right)^{1 /\left(1+4 p_{5}\right)}$. For the existence of de Sitter solutions the following relations hold between the coefficients

$$
\begin{aligned}
& c_{2}=\frac{3}{2}\left(\frac{2}{x_{\mathrm{dS}}^{2}}\right)^{p}(2+3 \alpha-4 \beta), \\
& c_{3}=\frac{\sqrt{2}}{2 p+2 q-1}\left(\frac{2}{x_{\mathrm{dS}}^{2}}\right)^{p+q}[p+3(p+q)(\alpha-\beta)],
\end{aligned}
$$

where $x_{\mathrm{dS}}=\dot{\phi}_{\mathrm{dS}} /\left(H_{\mathrm{dS}} M_{\mathrm{pl}}\right)$, and

$$
\begin{aligned}
& \alpha=\frac{4}{3}(2 p+4 q-1)\left(\frac{x_{\mathrm{dS}}^{2}}{2}\right)^{p+2 q} c_{4}, \\
& \beta=2 \sqrt{2}\left(p+3 q-\frac{1}{2}\right)\left(\frac{x_{\mathrm{dS}}^{2}}{2}\right)^{p+3 q} c_{5} .
\end{aligned}
$$

The background equations on the flat FLRW background are given by Eqs. (16) and (17), with different forms of $\rho_{\mathrm{DE}}$ and $P_{\mathrm{DE}}$ [65, 66]. Introducing the dimensionless variables $r_{1}=\left(\dot{\phi}_{\mathrm{dS}} / \dot{\phi}\right)^{2 q} H_{\mathrm{dS}} / H$ and $r_{2}=\left[\left(\dot{\phi} / \dot{\phi}_{\mathrm{dS}}\right)^{4} / r_{1}\right]^{(p+2 q) /(1+2 q)}$, the tracker solution corresponds to $r_{1}=1$ with the dark energy density parameter $\Omega_{\mathrm{DE}} \equiv \rho_{\mathrm{DE}} /\left(3 H^{2} M_{\mathrm{pl}}^{2}\right)=r_{2}$. After the radiation era the dark energy equation of state $w_{\mathrm{DE}}=P_{\mathrm{DE}} / \rho_{\mathrm{DE}}$ along the tracker is given by [65, 66]

$$
w_{\mathrm{DE}}=-\frac{1+s}{1+s \Omega_{\mathrm{DE}}}, \quad \text { where } \quad s=\frac{p}{2 q} .
$$

During the matter-dominated epoch $\left(\Omega_{\mathrm{DE}} \ll 1\right)$, it follows that $w_{\mathrm{DE}} \simeq-1-s$. The covariant Galileon corresponds to $s=1$ and $w_{\mathrm{DE}} \simeq-2$, in which case the tracker is incompatible with the observational data at the background level [61]. In the extended Galileon model it is possible to realize $w_{\mathrm{DE}}$ close to -1 for $s \ll 1$. The joint data analysis of SN Ia, CMB, and BAO shows that the parameter $s$ is constrained to be 66.

$$
s=0.034_{-0.034}^{+0.327} \quad(95 \% \mathrm{CL}) .
$$

Hence the tracker solution in the range $-1.36<w_{\mathrm{DE}}<$ -1 can be consistent with the background cosmology.

We choose the initial conditions $r_{1}=1$ and $r_{2}=$ $\Omega_{\mathrm{DE}} \ll 1$ in the early matter era. The dark energy density parameter $\Omega_{\mathrm{DE}}^{(0)}$ at the present epoch determines the initial value of $r_{2}$ at a given starting redshift. For given values of $p$ and $q$ there are two parameters $\alpha$ and $\beta$ left for the likelihood analysis in Sec. IV.

\section{COSMOLOGICAL PERTURBATIONS}

\section{A. Linear Perturbation Equation}

In Ref. [17 the full linear perturbation equations were derived for the most general scalar-tensor theories with second-order field equations [18, 19]. In this section we study the evolution of the cosmic growth rate in $f(R)$ gravity, covariant Galileon, and extended Galileon.

We consider the scalar metric perturbations $\Psi$ and $\Phi$ in the longitudinal gauge about the flat FLRW background. The perturbed line element is then given by 72

$$
d s^{2}=-(1+2 \Psi) d t^{2}+a^{2}(t)(1+2 \Phi) d \boldsymbol{x}^{2} .
$$

The energy density of non-relativistic matter is decomposed into the background and inhomogeneous parts as $\rho_{m}(t)+\delta \rho_{m}(t, \boldsymbol{x})$. We write the four-velocity of nonrelativistic matter in the form $u^{\mu}=\left(1-\Psi, \nabla^{i} v\right)$, where $v$ is the rotational-free velocity potential. We also introduce the following quantities:

$$
\delta \equiv \delta \rho_{m} / \rho_{m}, \quad \theta \equiv \nabla^{2} v
$$

In Fourier space with the comoving wavenumber $\boldsymbol{k}$, the matter perturbation obeys the following equations of motion:

$$
\begin{aligned}
& \dot{\delta}+\theta / a+3 \dot{\Phi}=0, \\
& \dot{\theta}+H \theta-\left(k^{2} / a\right) \Psi=0,
\end{aligned}
$$

where $k=|\boldsymbol{k}|$. We introduce the gauge-invariant density contrast

$$
\delta_{m} \equiv \delta+\left(3 a H / k^{2}\right) \theta .
$$

From Eqs. (38) and (39) it follows that

$$
\ddot{\delta}_{m}+2 H \dot{\delta}_{m}+\left(k^{2} / a^{2}\right) \Psi=3(\ddot{I}+2 H \dot{I}),
$$


where $I \equiv\left(a H / k^{2}\right) \theta-\Phi$.

In order to confront the models with galaxy clustering surveys, we are interested in the modes deep inside the Hubble radius. In this case we can employ the quasistatic approximation on sub-horizon scales, under which the dominant contributions to the perturbation equations are those including the terms $k^{2} / a^{2}, \delta$, and the mass $M$ of a scalar degree of freedom [55, 73]. Under this approximation we obtain the modified Poisson equation

$$
\left(k^{2} / a^{2}\right) \Psi \simeq-4 \pi G_{\text {eff }} \rho_{m} \delta,
$$

where $G_{\text {eff }}$ is the effective gravitational coupling whose explicit form is different depending on the theories [17]. In the framework of General Relativity, $G_{\text {eff }}$ is equivalent to the gravitational constant $G=1 /\left(8 \pi M_{\mathrm{pl}}^{2}\right)$.

Under the quasi-static approximation on sub-horizon scales, the r.h.s. of Eq. (41) can be neglected relative to the l.h.s. of it. Since $\delta_{m} \simeq \delta$, the matter perturbation obeys the following equation:

$$
\ddot{\delta}_{m}+2 H \dot{\delta}_{m}-4 \pi G_{\text {eff }} \rho_{m} \delta_{m} \simeq 0 .
$$

From this equation it is possible to predict $\delta_{m}, f_{m}$, and their evolution, and then it can be compared with the observed data of $f_{m}(z) \sigma_{8}(z)$.

\section{B. Comparison with Observations}

The perturbation $\delta_{g}$ of galaxies is related to $\delta_{m}$ via the bias factor $b$, i.e., $\delta_{g}=b \delta_{m}$. The galaxy power spectrum $\mathcal{P}_{g}^{s}(\boldsymbol{k})$ in the redshift space can be modeled as [21, 24]

$$
\mathcal{P}_{g}^{s}(\boldsymbol{k})=\mathcal{P}_{g g}(\boldsymbol{k})-2 \mu^{2} \mathcal{P}_{g \theta}(\boldsymbol{k})+\mu^{4} \mathcal{P}_{\theta \theta}(\boldsymbol{k}),
$$

where $\mu=\boldsymbol{k} \cdot \boldsymbol{r} /(k r)$ is the cosine of the angle of the $\boldsymbol{k}$ vector to the line of sight (vector $\boldsymbol{r}) . \mathcal{P}_{g g}(\boldsymbol{k})$ and $\mathcal{P}_{\theta \theta}(\boldsymbol{k})$ are the real space power spectra of galaxies and $\tilde{\theta}$, respectively, and $\mathcal{P}_{g \theta}(\boldsymbol{k})$ is the cross power spectrum of galaxy$\tilde{\theta}$ fluctuations in real space, where $\tilde{\theta}$ is related to $\theta$ but normalized by the Hubble velocity, i.e., $\tilde{\theta}=\theta /(a H)$.

For the linearly evolving perturbations Eq. (38) shows that $\theta$ is related to the growth rate of matter perturbations, as

$$
\begin{aligned}
\tilde{\theta} & =\theta /(a H) \simeq-f_{m} \delta_{m}=-f_{m} \delta_{g} / b, \\
f_{m} & \equiv \dot{\delta}_{m} /\left(H \delta_{m}\right),
\end{aligned}
$$

where we neglected the $\dot{\Phi}$ term. In this limit we obtain the famous Kaiser's formula [21]:

$$
\mathcal{P}_{g}^{s}(\boldsymbol{k})=\left(1+\mu^{2} \beta\right)^{2} \mathcal{P}_{g g}(\boldsymbol{k})
$$

where $\beta \equiv f_{m} / b$ is an anisotropy parameter. Therefore, the anisotropy of the measured power spectrum induced by RSD primarily constrains $\beta$, and we can measure the structure growth rate $f_{m}$ if $b$ is independently measured. When the independent $b$ measurement is not available, still we can measure the parameter combination $f_{m} \sigma_{8}$ relatively well, because the amplitude of the observed galaxy power spectrum is proportional to $\left(b \sigma_{8}\right)^{2}$, and hence $b$-dependence can be removed by taking the combination of the two observables in a galaxy redshift survey, as $\beta\left(\mathcal{P}_{g}^{s}\right)^{1 / 2} \propto f_{m} \sigma_{8}$.

There are some works in which nonlinear corrections, such as those coming from the velocity distribution of galaxies in collapsed structures, are added to the Kaiser's formula (47) 27]. In our work, we focus on the analysis in the linear regime $\left(k^{-1}>0.2 h^{-1} \mathrm{Mpc}\right)$ where nonlinear corrections are small. However, it is potentially important to fully take into account such effects for constraining dark energy models more precisely.

For a given model we can numerically solve Eq. (43) to find $\delta_{m}(z)$ and $\dot{\delta}_{m}(z)$ at a given scale of $x h^{-1}$ Mpc. In GR the cosmic growth rate is independent of scales for linear perturbations, but in some modified gravity models like $f(R)$ gravity the scale dependence is present even at the linear level. For the models close to the $\Lambda \mathrm{CDM}$ in the early cosmological epoch, the evolution of matter perturbations during the deep matter era is given by $\delta_{m} \propto a$, which corresponds to $\dot{\delta}_{m}=H \delta_{m}$ and hence $f_{m}=1$. The modification of gravity manifests itself in the late cosmological epoch.

Now it is easy to predict $f_{m}(z) \sigma_{x}(z)$ once the normalization of $\sigma_{x}$ is fixed, where $\sigma_{x} \propto \delta_{m}$ is the rms fluctuation amplitude at this scale. This is the quantity that we want to compare with the measurements of $f_{m}(z) \sigma_{8}(z)$. The quantity $f_{m} \sigma_{8}$ measured from a galaxy redshift survey is based on $\beta$ and $\mathcal{P}_{g}^{s}$, and hence it should essentially be regarded as $f_{m} \sigma_{x}$ with $x$ being the scale observed by the survey. The conversion into the conventional parameter $\sigma_{8}$ is done by assuming the standard shape of the linear matter power spectrum. Therefore, provided that a given modified gravity model predicts a matter power spectrum similar to that of $\Lambda \mathrm{CDM}$ at $z=0$, we can compare the theoretically predicted $f_{m}(z) \sigma_{x}(z)$ with the observed $f_{m}(z) \sigma_{8}(z)$, if we choose $x$ as the relevant galaxy survey scale and normalize $\sigma_{x}(z)$ as $\sigma_{x}(z=0)=\sigma_{8}(z=0)$. For this normalization, we use the WMAP7 year bound [74]

$$
\sigma_{8}(z=0)=0.811_{-0.031}^{+0.030},
$$

which primarily comes from the amplitude at the recombination. We note that this value is derived by assuming the $\Lambda$ CDM model. Therefore, we also take into account the following bound constrained from clustering of galaxies and galaxy clusters [75]:

$$
\sigma_{8}(z=0)=0.795_{-0.030}^{+0.030} .
$$

In our likelihood analysis we take a slightly wider range $0.75<\sigma_{8}(z=0)<0.85$ than those constrained above.

The data of $f_{m} \sigma_{8}$ used to constrain the models are summarized in Table \. We treat these data as independent measurements, and calculate statistically allowed model parameter space by the standard $\chi^{2}$ analysis. This 


\begin{tabular}{lccc}
\hline \hline \multicolumn{1}{c}{$z$} & $f_{m}(z) \sigma_{8}(z)$ & $1 / k\left[h^{-1} \mathrm{Mpc}\right]$ & Survey \\
\hline 0.067 & $0.423 \pm 0.055$ & $16-30$ & 6dFGRS $(2012)[30]$ \\
0.17 & $0.51 \pm 0.06$ & $6.7-50$ & 2dFGRS (2004) [23] \\
0.22 & $0.42 \pm 0.07$ & $3.3-50$ & WiggleZ (2011) [27] \\
0.25 & $0.3512 \pm 0.0583$ & $30-200$ & SDSS LRG (2011) [28] \\
0.37 & $0.4602 \pm 0.0378$ & $30-200$ & SDSS LRG (2011) [28] \\
0.41 & $0.45 \pm 0.04$ & $3.3-50$ & WiggleZ (2011) [27] \\
0.57 & $0.415 \pm 0.034$ & $25-160$ & BOSS CMASS (2012) [29] \\
0.6 & $0.43 \pm 0.04$ & $3.3-50$ & WiggleZ (2011) [27] \\
0.78 & $0.38 \pm 0.04$ & $3.3-50$ & WiggleZ (2011) [27] \\
\hline \hline
\end{tabular}

Table I: Data of $f_{m} \sigma_{8}$ measured from RSD with the survey references.

is justified for different surveys in different volumes, and also approximately for different redshift bins in one survey, since RSD is mainly measured by the Fourier modes whose scale is smaller than the redshift bin size. There is another constraint $f_{m} \sigma_{8}=0.49 \pm 0.18$ at $z=0.77$ derived by Guzzo et al. [26], but we exclude this data because of more recent WiggleZ measurement at the redshift $z=0.78$ [27] (see Ref. [31]).

The ranges of scales relevant to these surveys are also given in Table【 It should be noted that, even though we compare the theory and data in terms of $f_{m} \sigma_{8}$, we are essentially comparing $f_{m} \sigma_{x}$, under the condition that the mapping between $\sigma_{8}$ and $\sigma_{x}$ in modified gravity models is similar to that in the concordance cosmological model.

It should be noted that the actual measurements of $f_{m} \sigma_{8}$ should be affected by systematic errors coming from the nonlinear effects, which are ignored in the simple Kaiser formula. Such systematic uncertainties are extensively discussed in the experimental papers deriving $f_{m} \sigma_{8}$ such as WiggleZ [27] and BOSS [29]. It is generally believed that such systematic errors are smaller than the statistical errors of currently available surveys, and hence we simply adopt the $f_{m} \sigma_{8}$ measurements reported in the observational papers.

Based on the WMAP7 [74] constraint on the today's density parameter of dark energy

$$
\Omega_{\mathrm{DE}}^{(0)}=0.725 \pm 0.016
$$

we adopt $\Omega_{\mathrm{DE}}^{(0)}=0.725$ as the standard value in this paper, but we also test the dependence on this parameter within the range of $0.70 \leq \Omega_{\mathrm{DE}}^{(0)} \leq 0.75$, which is slightly wider than the $1 \sigma$ error of WMAP7. Below we discuss the evolution of perturbations in three modified gravity models separately, and compare them with the observed data for some representative parameter sets.

\section{C. $f(R)$ gravity}

The effective gravitational coupling in metric $f(R)$ gravity is given by [55]

$$
G_{\text {eff }}=\frac{G}{F} \frac{1+4 m(k / a)^{2} / R}{1+3 m(k / a)^{2} / R},
$$

where the parameter $m \equiv R F_{, R} / F$ characterizes the deviation from the $\Lambda \mathrm{CDM}$ model $(f=R-2 \Lambda)$. In order to avoid ghosts and tachyonic instabilities we require $F>0$ and $F_{, R}>0$ (with $R>0$ ), respectively [53], so that $m>0$.

Equation (51) shows that the transition from the "General Relativistic regime" ( $\left.G_{\text {eff }}=G / F\right)$ to the "modified gravitational regime" $\left(G_{\text {eff }}=4 G /(3 F)\right)$ occurs around $m \approx(a H / k)^{2}$. If such a transition occurs at the redshift $z_{c}$ larger than 1 , the transition redshift for the model (6) can be estimated as [76]

$$
z_{c} \approx\left[\left(\frac{k}{a_{0} H_{0}}\right)^{2} \frac{2 n(2 n+1)}{\lambda^{2 n}} \frac{\left(2 \Omega_{\mathrm{DE}}^{(0)}\right)^{2 n+1}}{\left.\Omega_{m}^{(0)}\right)^{2(n+1)}}\right]^{1 /(6 n+4)}-1,
$$

where $a_{0}$ is the today's scale factor normalized as $a_{0}=1$, $\Omega_{\mathrm{DE}}^{(0)}$ and $\Omega_{m}^{(0)}$ are the today's density parameters of dark energy and non-relativistic matter respectively. We identify the present epoch to be $\Omega_{\mathrm{DE}}^{(0)}=0.72$. The modes with larger $k$ enter the modified gravitational regime earlier, so that the growth of perturbations tends to be more significant. For smaller $n$ and $\lambda$, the transition redshift also gets larger. Recall that $n$ and $\lambda$ are bounded from below from Eqs. (7)-(9).

Numerically we solve the perturbation equations without using the quasi-static approximation on sub-horizon scales. The initial conditions of the perturbation $\delta R$ is chosen such that the oscillating mode is sub-dominant relative to the matter-induced mode (along the lines of Refs. [53, 55]). The quasi-static approximation based on Eq. (43) with the gravitational coupling (51) is accurate enough to estimate the growth rate of perturbations for sub-horizon modes [77, 78].

In Fig. 1 the evolution of $f_{m} \sigma_{8}$ is plotted for $n=1$ and three different values of $\lambda$, with the three different wavenumbers of $k^{-1}=10,30$, and $60 h^{-1} \mathrm{Mpc}$ within the range of galaxy survey scales. When $\lambda=1.55$ the numerical value of the transition redshift is larger than a few and hence the effective gravitational coupling in the regime $z \lesssim 1$ is given by $G_{\text {eff }} \approx 4 G /(3 F)$. As we see in Fig. 11 $f_{m} \sigma_{8}$ is larger than that in the $\Lambda \mathrm{CDM}$ model. When $n=1$ and $\lambda=1.55$, the growth rate is almost independent of scales, which reflects the fact that $z_{c}$ is larger than 1 on those scales.

In Fig. 1 we find that the dispersion of $f_{m} \sigma_{8}$ with respect to $\lambda$ appears on larger scales. This comes from the fact that, for smaller $k$ and larger $\lambda$, the transition redshift (52) gets smaller. Even for the scale $60 h^{-1} \mathrm{Mpc}$ and $\lambda=20$, however, $f_{m} \sigma_{8}$ is larger than that in the $\Lambda \mathrm{CDM}$, 

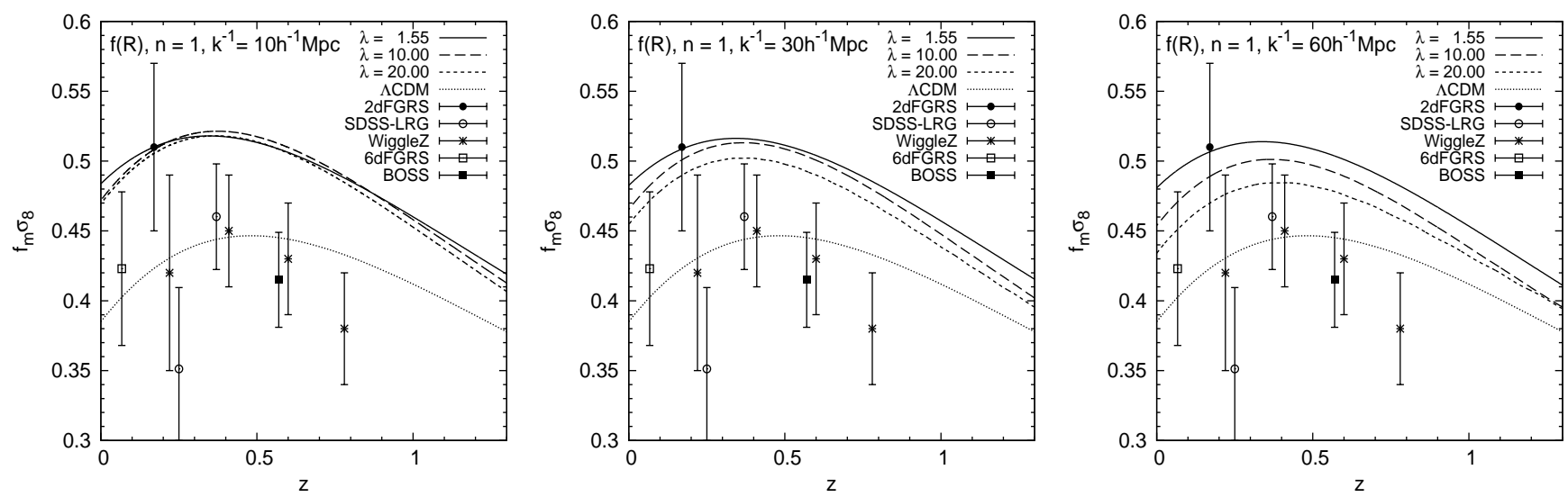

Figure 1: Evolution of $f_{m} \sigma_{8}$ versus the redshift $z$ for the $f(R)$ model (6) with $n=1, \sigma_{8}(z=0)=0.811$, and $\Omega_{\mathrm{DE}}^{(0)}=0.72$. The left, middle, and right panels correspond to the scales $k^{-1}=10 h^{-1}, 30 h^{-1}, 60 h^{-1} \mathrm{Mpc}$, respectively. The solid, long dashed, dashed curves correspond to $\lambda=1.55,10,20$, respectively, whereas the dotted curve corresponds to the $\Lambda$ CDM. We also plot the observational bounds on $f_{m} \sigma_{8}$ constrained from 2dFGRS [23], SDSS-LRG [28], WiggleZ [27], 6dFGRS [30], and BOSS [29].
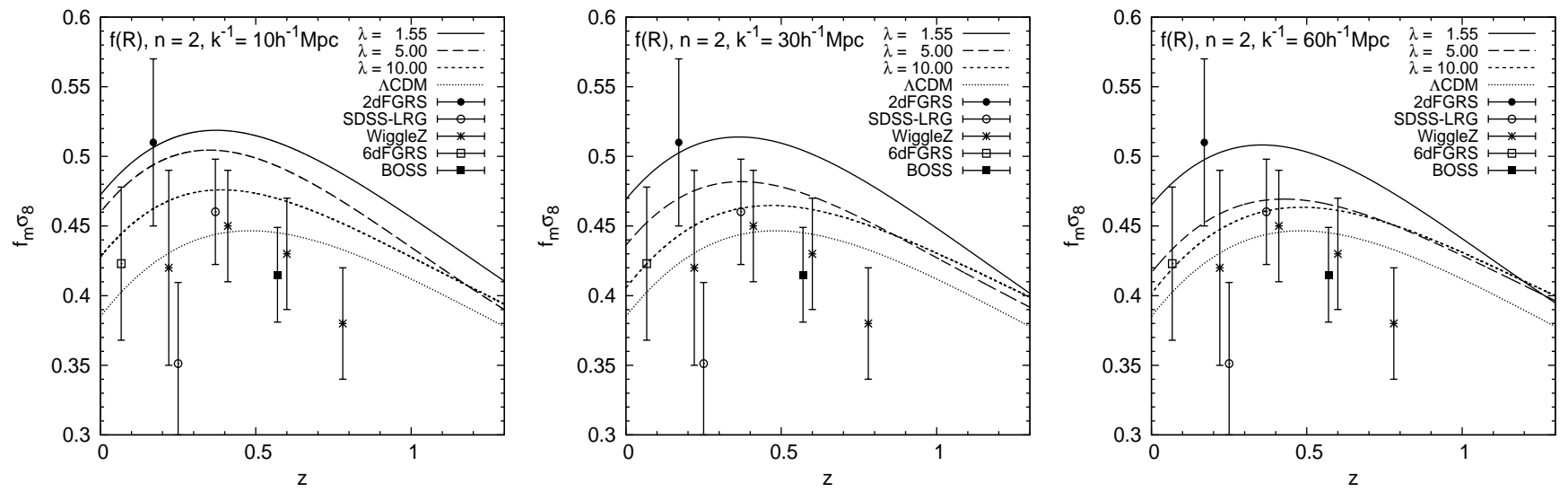

Figure 2: The same as Fig. 1] but for $n=2$ and $\lambda=1.55,5$, and 10 .

so that the $f(R)$ model with $n=1$ can be distinguished from the $\Lambda \mathrm{CDM}$.

For larger $n$ the transition redshift $z_{c}$ tends to be smaller, and the growth rate of $\delta_{m}$ today gets smaller for given $k$ and $\lambda$. In Fig. 2 we show the evolution of $f_{m} \sigma_{8}$ for $n=2$ and $\lambda=1.55,5$, and 10. In this case the dispersion of $f_{m} \sigma_{8}$ with respect to $\lambda$ appears even for the scale $10 h^{-1} \mathrm{Mpc}$. On the scales larger than $30 h^{-1} \mathrm{Mpc}$ the values of $f_{m} \sigma_{8}$ are almost degenerate for $\lambda \gtrsim 10$, whereas for $\lambda=\mathcal{O}(1)$ the growth rate is still large even on the scale $60 h^{-1}$ Mpc.

When compared with the observed data in Figs. 1 and 2. the $f(R)$ model (6) with $n$ and $\lambda$ of the order of 1 is in tension with most of the data. In Sec. IV] we place observational constraints on the model parameters $n$ and $\lambda$.

\section{Covariant Galileon}

The effective gravitational coupling for the covariant Galileon is given by [16] (see also Refs. [17, 79 81])

$$
G_{\mathrm{eff}}=\frac{2 M_{\mathrm{pl}}^{2}\left(\mathcal{C}_{4}^{2}-\mathcal{C}_{3} \mathcal{C}_{5}\right)}{2 \mathcal{C}_{1} \mathcal{C}_{2} \mathcal{C}_{4}-\mathcal{C}_{1}^{2} \mathcal{C}_{5}-\mathcal{C}_{2}^{2} \mathcal{C}_{3}} G
$$

where the time-dependent coefficients $\mathcal{C}_{i}$ 's are

$$
\begin{aligned}
\mathcal{C}_{1}= & 2 M_{\mathrm{pl}}^{2}+3 c_{4} \dot{\phi}^{4} / M^{6}-6 c_{5} H \dot{\phi}^{5} / M^{9} \\
\mathcal{C}_{2}= & -c_{3} \dot{\phi}^{2} / M^{3}+12 c_{4} H \dot{\phi}^{3} / M^{6}-15 c_{5} H^{2} \dot{\phi}^{4} / M^{9},(55) \\
\mathcal{C}_{3}= & 2 M_{\mathrm{pl}}^{2}-c_{4} \dot{\phi}^{4} / M^{6}-6 c_{5} \dot{\phi}^{4} \ddot{\phi} / M^{9} \\
\mathcal{C}_{4}= & 12 c_{4} \dot{\phi}^{2} \ddot{\phi} / M^{6}+4 c_{4} H \dot{\phi}^{3} / M^{6}-6 c_{5} \dot{H} \dot{\phi}^{4} / M^{9} \\
& -6 c_{5} H^{2} \dot{\phi}^{4} / M^{9}-24 c_{5} H \dot{\phi}^{3} \ddot{\phi} / M^{9}
\end{aligned}
$$




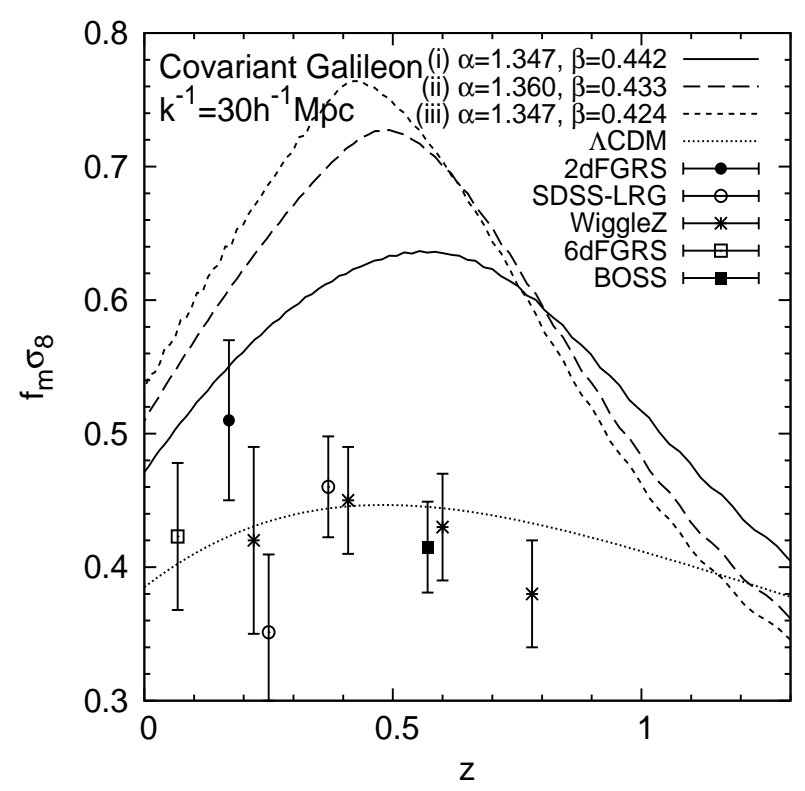

Figure 3: Evolution of $f_{m} \sigma_{8}$ versus the redshift $z$ for the scale $30 \mathrm{~h}^{-1} \mathrm{Mpc}$ in the covariant Galileon model with $\sigma_{8}(z=0)=$ 0.811 and $\Omega_{\mathrm{DE}}^{(0)}=0.73$. The solid, long dashed, and dashed curves correspond to the cases (i) $\alpha=1.347, \beta=0.442$, (ii) $\alpha=1.360, \beta=0.433$, and (iii) $\alpha=1.347, \beta=0.424$, respectively, whereas the dotted curve shows the evolution of $f_{m} \sigma_{8}$ in the $\Lambda$ CDM model. The observational data are the same as those plotted in Fig. 1.

$$
\begin{aligned}
\mathcal{C}_{5}= & c_{2}-4 c_{3} H \dot{\phi} / M^{3}-2 c_{3} \ddot{\phi} / M^{3}+26 c_{4} H^{2} \dot{\phi}^{2} / M^{6} \\
& +12 c_{4} \dot{H} \dot{\phi}^{2} / M^{6}+24 c_{4} H \dot{\phi} \ddot{\phi} / M^{6} \\
& -36 c_{5} H^{2} \dot{\phi}^{2} \ddot{\phi} / M^{9}-24 c_{5} H \dot{\phi}^{3}\left(H^{2}+\dot{H}\right) / M^{9} .(58)
\end{aligned}
$$

At the de Sitter solution $\left(r_{1}=r_{2}=1\right)$ the formula (53) reduces to

$$
\frac{G_{\mathrm{eff}}}{G}=\frac{1}{3(\alpha-2 \beta)}
$$

This shows that $\alpha-2 \beta>0$ to avoid ghosts. The latetime tracker corresponds to the solution along which the conditions $r_{1} \ll 1$ and $r_{2} \ll 1$ are satisfied during most of the cosmological epoch prior to the dominance of dark energy. In this regime, expansion of $G_{\text {eff }}$ around $r_{1}=0$ and $r_{2}=0$ gives

$$
\frac{G_{\text {eff }}}{G} \simeq 1+\left(\frac{255}{8} \beta+\frac{211}{16} \alpha r_{1}\right) r_{2} .
$$

The non-ghost condition corresponds to $\beta r_{2}>0$ [45], so that $G_{\text {eff }}>G$. Hence the growth rate of $\delta_{m}$ is larger than that in the $\Lambda \mathrm{CDM}$.

Numerically we solve the full perturbation equations presented in Refs. [16, 17] without using the quasistatic approximation for the model parameters given in Eq. (24). The quantity $r_{1}$ needs to be much smaller than
1 during the early matter era, in which case the solutions approach the tracker at late times. The initial conditions for the perturbations are chosen to satisfy $\dot{\Phi}=0$ and $\dot{\delta \phi}=0$ in the deep matter era (see Ref. [16] for detail).

In order to avoid ghosts and Laplacian instabilities the parameters $\alpha$ and $\beta$ are restricted to be in the range $0<$ $\alpha-2 \beta<2 / 3$ [45, 46]. Numerically we find that, for $\alpha-$ $2 \beta>0.505$, the perturbations show violent instabilities during the transition from the matter era to the de Sitter epoch. This is associated with the fact that the effective gravitational coupling (53) tends to cross 0 for larger $\alpha$. Hence we focus on the parameter space $0<\alpha-2 \beta<$ 0.505 , in addition to the constraints from the background evolution [Eq. (24)].

In Fig. 3 we plot the evolution of $f_{m} \sigma_{8}$ for the scale $k^{-1}=30 h^{-1} \mathrm{Mpc}$ with several different values of $\alpha$ and $\beta$. For larger values of $\alpha-2 \beta$ the growth of $\delta_{m}$ tends to be more significant, so that $f_{m} \sigma_{8}$ gets larger. Even in case (i) (see caption of Fig. 3), which gives the smallest $f_{m} \sigma_{8}$ within the allowed ranges of $\alpha$ and $\beta$, the model is in tension with the recent RSD data because of the large growth rate of $\delta_{m}$. Numerically we also confirm that the evolution of $f_{m} \sigma_{8}$ is practically independent of the scales in the range $10 h^{-1} \mathrm{Mpc} \lesssim k^{-1} \lesssim 60 h^{-1} \mathrm{Mpc}$.

Since the growth rate of linear perturbations is practically independent of scales, only the total amplitude of the matter power spectrum is subject to change relative to that in the $\Lambda \mathrm{CDM}$. The matter power spectra of covariant Galileon and $\Lambda$ CDM models were explicitly evaluated in Fig. 6 of Ref. [80], which shows the property mentioned above. Hence the mapping between $\sigma_{8}$ and $\sigma_{x}$ to derive the constraint on $f_{m} \sigma_{8}$ in the $\Lambda$ CDM model should be valid for the covariant Galileon model as well.

\section{E. Extended Galileon}

The effective gravitational coupling of for the extended Galileon is given by the form (53) with more complicated coefficients $\mathcal{C}_{i}$ (see Ref. 66] for details). At the de Sitter solution $\left(r_{1}=r_{2}=1\right)$ it follows that

$$
\frac{G_{\text {eff }}}{G}=\frac{2}{2(1-p)+3(1+2 q)(\alpha-2 \beta)} .
$$

The general expression of $G_{\text {eff }}$ is quite involved even along the tracker $\left(r_{1}=1\right)$. In the following we focus on the tracker solution $\left(r_{1}=1\right)$ for the model $p=1$ and $q=5 / 2$, in which case $s=0.2$ and hence $w_{\mathrm{DE}}=-1.2$ during the matter era. This model shows some deviation from the $\Lambda \mathrm{CDM}$ model, but it is still consistent with the background-level observations (see Eq. (35)). In the regime $r_{2} \ll 1, G_{\text {eff }}$ is approximately given by

$$
\begin{aligned}
\frac{G_{\mathrm{eff}}}{G} \simeq & 1+\left[48411 \alpha^{2}-3 \alpha(3560+60291 \beta)\right. \\
& \left.+22\left(50+924 \beta+7641 \beta^{2}\right)\right] r_{2} \\
& /[10(308-1536 \alpha+3201 \beta)],
\end{aligned}
$$




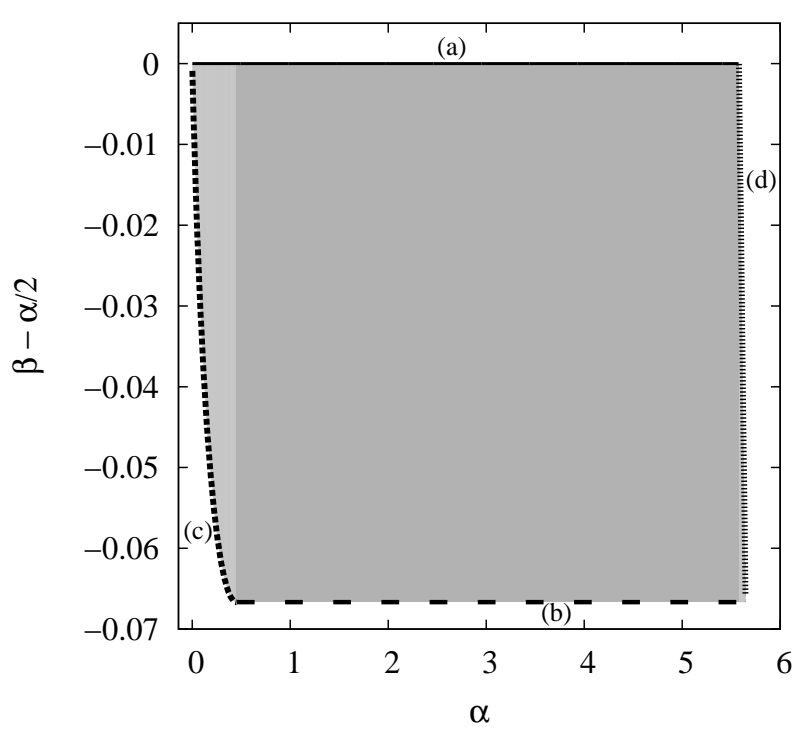

Figure 4: The grey region shows the theoretically allowed parameter space for the extended Galileon model with $p=1$ and $q=5 / 2$ (the region in which ghosts and Laplacian instabilities are absent). Each border corresponds to (a) $\beta=\alpha / 2$, (b) $\beta=\alpha / 2-1 / 15$, (c) $\beta=(408 \alpha+68-$ $2 \sqrt{17} \sqrt{3(272-75 \alpha) \alpha+68}) / 561$, and (d) $\beta=(242-15 \alpha+$ $4 \sqrt{3630-495 \alpha}) / 99$, respectively. Taken from Ref. 66].

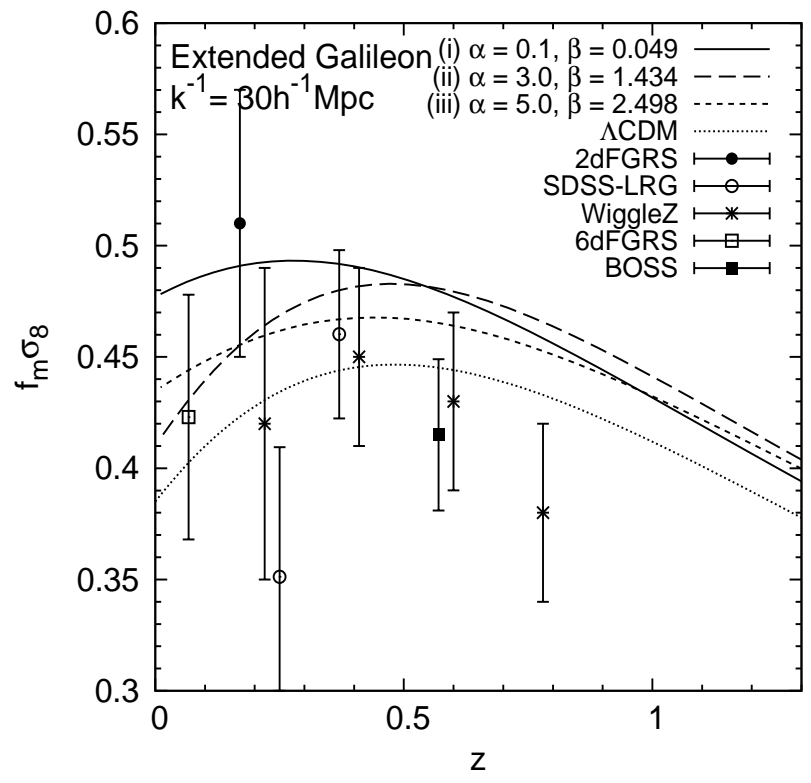

Figure 5: Evolution of $f_{m} \sigma_{8}$ versus the redshift $z$ for the scale $30 h^{-1} \mathrm{Mpc}$ in the extended Galileon model with $\sigma_{8}(z=0)=$ 0.811 and $\Omega_{\mathrm{DE}}^{(0)}=0.72$. The solid, long dashed, and dashed curves correspond to cases (i) $\alpha=0.1, \beta=0.049$, (ii) $\alpha=3$, $\beta=1.434$, and (iii) $\alpha=5, \beta=2.498$, respectively, whereas the dotted curve corresponds to the $\Lambda$ CDM. The observational data are the same as those plotted in Fig. 1 whereas at the de Sitter solution $G_{\text {eff }} / G=1 /[9(\alpha-2 \beta)]$.

In Refs. [65, 66] the authors clarified the parameter space in which ghosts and Laplacian instabilities of scalar and tensor perturbations are absent. Figure 4 shows such a viable region, which is surrounded by the 4 borders given in the caption. On the line (a) $\beta=\alpha / 2$, one has $G_{\text {eff }} / G \rightarrow \infty$ at the de Sitter solution and $G_{\text {eff }} / G \simeq$ $1+4(275-129 \alpha) r_{2} /[5(616+129 \alpha)]$ for $r_{2} \ll 1$. In the latter regime $G_{\text {eff }}$ gets larger for smaller $\alpha$. Hence today's values of $f_{m} \sigma_{8}$ on the line (a) are large around the region $\alpha=\beta=0$. The case (i) in Fig. 5 corresponds to such an example.

On the line (b) shown in Fig. 4, we have $G_{\text {eff }} / G=5 / 6$ at the de Sitter solution and $G_{\text {eff }} / G \simeq 1+13 r_{2} / 25$ for $r_{2} \ll 1$. In the regime $r_{2} \ll 1, G_{\text {eff }}$ on the line (b) is larger than that on the line (a) for the same value of $\alpha$, but at the de Sitter solution $G_{\text {eff }}$ is even smaller than that for the $\Lambda$ CDM. The case (ii) in Fig. [5]corresponds to such an example, which exhibits rapid decrease of $f_{m} \sigma_{8}$ for $z<0.5$. This case shows a better compatibility with the RSD data in the low redshift regime relative to the case (i).

Around the line (a), the effective gravitational coupling $G_{\text {eff }}$ in the regime $r_{2} \ll 1$ is smaller than $G$ for $\alpha>$ $275 / 129$. Although $G_{\text {eff }}$ goes to infinity at the de Sitter solution, the growth rates of matter perturbations are suppressed in high redshifts for $\alpha>275 / 129$. The case (iii) in Fig. 5 corresponds to such an example, which is consistent with some of the RSD data because of the suppression of $f_{m} \sigma_{8}$ in the early cosmological epoch.

We also confirm that the evolution of $f_{m} \sigma_{8}$ is practically independent of the scales in the range $10 h^{-1} \mathrm{Mpc}$ $\lesssim k^{-1} \lesssim 60 h^{-1} \mathrm{Mpc}$.

\section{OBSERVATIONAL CONSTRAINTS FROM RSD}

Here we determine quantitative model parameter space that is excluded from the RSD data for the three modified gravity models discussed in Sec. III The confidence regions of excluded parameter spaces are calculated by the standard $\chi^{2}$ analysis. For a given set of model parameters, $\chi_{\mathrm{obs}}^{2}$ is calculated from the 9 data points of $f_{m}(z) \sigma_{8}(z)$, and the exclusion confidence level at this point is the probability of $\chi^{2}<\chi_{\text {obs }}^{2}$ for the $\chi^{2}$ distribution with $d$ degrees of freedom, where $d=N_{d}-N_{m}$, $N_{d}=9$ is the data number, and $N_{m}$ is the number of model parameters. For all the three modified gravity models considered here, $N_{m}=2$.

In three classes of modified gravity models studied below, we first carry out the likelihood analysis by fixing $\sigma_{8}(z=0)=0.811$. We then discuss the cases in which $\sigma_{8}(z=0)$ is varied in the range $0.75<\sigma_{8}(z=0)<0.85$. We also fix the value of $\Omega_{\mathrm{DE}}^{(0)}$ to be 0.725 constrained by the background cosmology first and then check whether the constraints are subject to change by varying $\Omega_{\mathrm{DE}}^{(0)}$ in 
the range $0.70<\Omega_{\mathrm{DE}}^{(0)}<0.75$.

\section{A. $\quad f(R)$ model}

We first place observational constraints on the $f(R)$ model (6) for three different scales $10 h^{-1}, 30 h^{-1}$, and $60 h^{-1} \mathrm{Mpc}$. We identify the present epoch to be $\Omega_{\mathrm{DE}}^{(0)}=$ 0.72 with the amplitude $\sigma_{8}(z=0)=0.811$. In Fig. 6] we plot the parameter space in the $(n, \lambda)$ plane constrained by the RSD data shown in Table [1. The grey regions are forbidden by the violations of the de Sitter stability condition (8) as well as that of the local gravity constraint (9). Figure 6 shows that observational constraints tend to be tighter for smaller scales.

The model with $n=1$ and $\lambda<20$ is outside the $95.4 \%$ CL contour for the scales $10 h^{-1} \mathrm{Mpc} \lesssim k^{-1} \lesssim$ $60 h^{-1} \mathrm{Mpc}$. This reflects the fact that the cosmic growth rates in this parameter space are too large to be compatible with the recent $\mathrm{RSD}$ data in the regime $z \lesssim 1$, irrespective of the values of $\lambda$ (see Fig. 11).

For $n=2$, the parameter $\lambda$ is constrained to be $\lambda>8\left(10 h^{-1} \mathrm{Mpc}\right), \lambda>5\left(30 h^{-1} \mathrm{Mpc}\right)$, and $\lambda>3$ $\left(60 h^{-1} \mathrm{Mpc}\right)$ at $95.4 \% \mathrm{CL}$. In terms of the dimensionless parameter $m=R F_{, R}(R) / F(R)$ characterizing the deviation from the $\Lambda$ CDM 70 , we have $m(z=0)=9.6 \times 10^{-5}$ for $n=2, \lambda=8$ and $m(z=0)=6.3 \times 10^{-4}$ for $n=2, \lambda=5$. Hence, for $n=2$, today's values of $m$ are bounded to be $m(z=0)<9.6 \times 10^{-5}\left(10 h^{-1} \mathrm{Mpc}\right)$ and $m(z=0)<6.3 \times 10^{-4}\left(30 h^{-1} \mathrm{Mpc}\right)$ at $95.4 \%$ CL. The constraint on $m(z=0)$ coming from the integrated Sachs-Wolfe effect of the CMB anisotropies is much weaker, $m(z=0) \lesssim 1[6,40]$. This shows that the measurement of the cosmic growth rate from RSD is sufficiently powerful to constrain the $f(R)$ gravity model tightly.

If we choose the values of $n$ greater than 3 , the model is close to the $\Lambda$ CDM. In this case, however, the scalaron mass is very heavy in the early cosmological epoch, so that there is a severe fine tuning for the initial conditions of perturbations to avoid the dominance of the oscillating mode [53, 55]. Hence it is not so meaningful to consider such cases even if $\chi^{2}$ may be reduced relative to the case $n \lesssim 2.5$.

In addition to two-dimensional constraints, we carry out the likelihood analysis by fixing one of the parameters $n$ and $\lambda$. The results of such one-dimensional constraints are summarized in Table II. For larger values of $n$ (or $\lambda)$, the allowed region of the other parameter tends to be wider.

We also study how the two-dimensional constraints are subject to change when $\sigma_{8}(z=0)$ and $\Omega_{\mathrm{DE}}^{(0)}$ vary. For larger $\sigma_{8}(z=0)$ and smaller $\Omega_{\mathrm{DE}}^{(0)}$ the observational contours shift the region of smaller values of $n$ and $\lambda$, so that the constraints get tighter. We confirm that the essential result does not change by varying these parameters in the range $0.75<\sigma_{8}(z=0)<0.85$ and $0.70<\Omega_{\mathrm{DE}}^{(0)}<0.75$.

\begin{tabular}{ccc}
\hline \hline Theory & Fixed parameter & Constraint \\
\hline$f(R)$ & $n=1.0$ & $\lambda \gg 20$ \\
& $n=1.5$ & $\lambda>9.7$ \\
& $n=2.0$ & $\lambda>4.6$ \\
& $\lambda=1.55$ & $n \gg 2.5$ \\
& $\lambda=5.0$ & $n>1.92$ \\
& $\lambda=10$ & $n>1.48$ \\
Extended & $\alpha=0.1$ & $-0.061<\beta-\alpha / 2<-0.039$ \\
Galileon & $\alpha=3.0$ & $-0.063<\beta-\alpha / 2<0$ \\
$(s=0.2)$ & $\alpha=5.0$ & $-0.063<\beta-\alpha / 2<0$ \\
& $\beta-\alpha / 2=-0.01$ & $\alpha>1.0$ \\
& $\beta-\alpha / 2=-0.02$ & $\alpha>0.7$ \\
& $\beta-\alpha / 2=-0.066$ & no allowed region \\
\hline \hline
\end{tabular}

Table II: One-dimensional constraints on $f(R)$ and extended Galileon theories $(95.4 \% \mathrm{CL})$. The other parameters are fixed to be $k^{-1}=30 h^{-1} \mathrm{Mpc}, \sigma_{8}(z=0)=0.811$, and $\Omega_{\mathrm{DE}}^{(0)}=$ 0.72 . " $\gg$ " means that there are no allowed regions within our calculation range. Covariant Galileon theory has no allowed parameter space at $95.4 \%$ CL.

In Refs. 43] it was pointed out that non-linear perturbations in $f(R)$ gravity start to provide some contribution to the redshift-space power spectrum even for $k=0.02 h \mathrm{Mpc}^{-1}$ (see Fig. 4 of [43]). The non-linear effect suppresses the redshift-space power spectrum especially for $k \gtrsim 0.1 h \mathrm{Mpc}^{-1}$. It remains to be seen how the observational constraints are subject to change by non-linear perturbations.

\section{B. Covariant Galileon}

For the covariant Galileon the allowed parameter space is constrained as Eq. (24) from the combined data analysis of SN Ia (Union2), CMB, and BAO. If the Constitution SN Ia data are used instead of Union2, the observational bounds on $\alpha$ and $\beta$ become $\alpha=1.411 \pm 0.056$ and $\beta=0.422 \pm 0.022[61]$. Setting the prior $\alpha-2 \beta<0.505$ to avoid the negative values of $G_{\text {eff }}$, the allowed parameter space is the triangle surrounded by the three points $(\alpha, \beta)=$ $(1.347,0.421),(1.347,0.442),(1.389,0.442)$ for Union2 and $(\alpha, \beta)=(1.355,0.425),(1.355,0.444),(1.393,0.444)$ for Constitution. In these parameter regions we further place constraints on the model from the RSD data.

In Fig. 7 we show observational bounds on the covariant Galileon model in the $(\alpha, \beta)$ plane for the scale $30 h^{-1} \mathrm{Mpc}$ with $\sigma_{8}(z=0)=0.811$ and $\Omega_{\mathrm{DE}}^{(0)}=0.73$. The parameter space constrained from the background cosmology is excluded at more than $11 \sigma$ CL. This comes from the large cosmic growth rate relative to that of the $\Lambda C D M$, as shown in Fig. 3. As the model parameters approach the border $\alpha-2 \beta=0.505$, it is further difficult to match with the data because of the significant enhancement of matter perturbations. We also find that the observational constraints are practically independent 

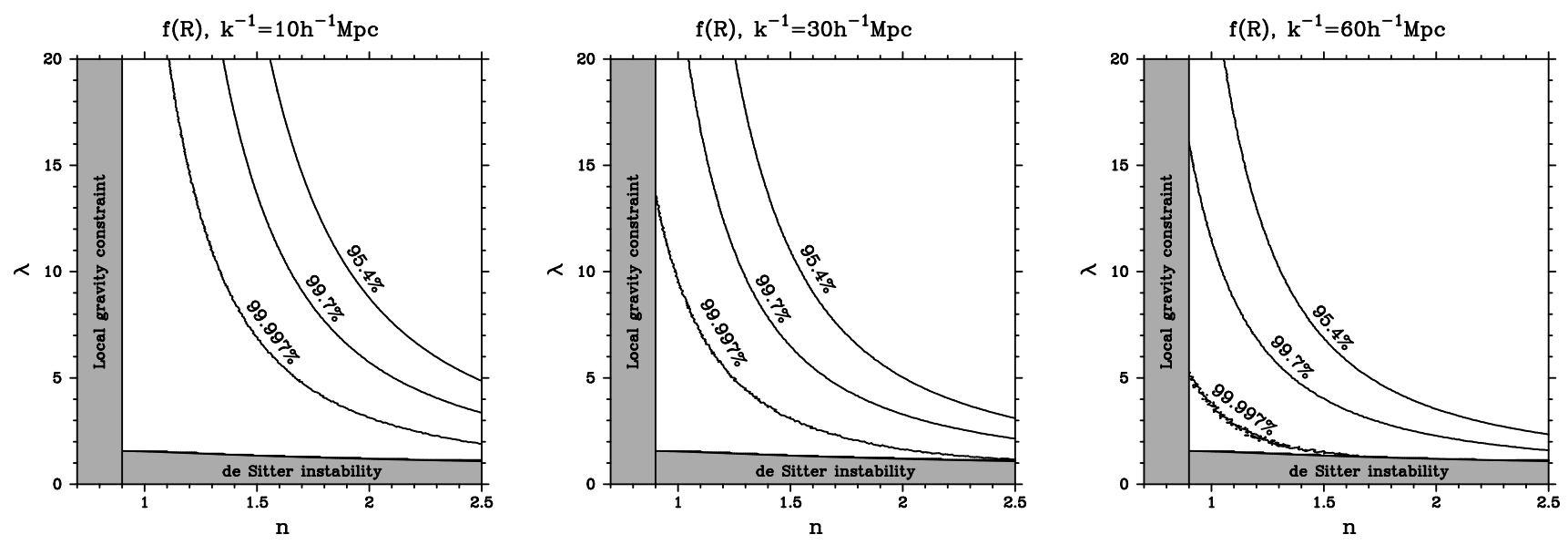

Figure 6: Constraints on the Hu-Sawicki $f(R)$ gravity model [6] from the RSD data in the $(n, \lambda)$ plane with $\sigma_{8}(z=0)=0.811$ and $\Omega_{\mathrm{DE}}^{(0)}=0.72$ for three different scales of $k^{-1}=10 h^{-1} \mathrm{Mpc}$ (left), $30 h^{-1}$ (middle), $60 h^{-1}$ Mpc (right). The lower-left regions are excluded by the confidence levels indicated along with the contours.

of the scales. For the scale $60 h^{-1} \mathrm{Mpc}$ the covariant Galileon is excluded at more than $10 \sigma$ CL.

A similar conclusion was recently reached in Ref. [47, in which the authors included the WiggleZ and BOSS data as well as SN Ia, CMB, BAO data. The difference from their work is that we have taken into account the 2dFGRS, 6dFGRS, and SDSS-LRG data as well. Even though the 2dFGRS and 6dFGRS data provide larger values of $f_{m} \sigma_{8}$ relative to those from WiggleZ and BOSS, the covariant Galileon cannot be consistent with most of the currently available RSD data.

For smaller values of $\sigma_{8}(z=0)$, the constraint becomes looser. In the range $0.75<\sigma_{8}(z=0)<0.85$, however, the whole parameter space is still rejected at least at $8 \sigma$ CL. If we take much smaller $\sigma_{8}(z=0)$ such as 0.6 , there could be some allowed region at $95.4 \%$ CL. However, such low values of $\sigma_{8}(z=0)$ are disfavored from the constraint from clustering of galaxies [75].

\section{Extended Galileon}

In the extended Galileon model with $p=1$ and $q=$ $5 / 2$, the theoretically allowed parameter space is given by the region shown in Fig. (1. As mentioned in the previous section, this plot assumes that the dark energy equation of state during the matter era is $w_{\mathrm{DE}}=-1-s=-1.2$, where $s=p /(2 q)$. This model is interesting because it is different from the $\Lambda \mathrm{CDM}$ model but is still consistent with the joint constraint of SN Ia, CMB, and BAO [66] (see also Eq. (35)).

In Fig. 8 we plot the observational bounds in the $(\alpha, \beta)$ plane for the scale $30 h^{-1} \mathrm{Mpc}$ with $\sigma_{8}(z=0)=0.811$ and $\Omega_{\mathrm{DE}}^{(0)}=0.72$ (in which case the dark energy density parameter is consistent with the background constraint derived in Ref. 66] ). For the scales ranging $10 h^{-1} \mathrm{Mpc}$ $\lesssim k^{-1} \lesssim 60 h^{-1}$ Mpc we confirm that the constraints are similar to those shown in Fig. 8. Unlike the covariant
Galileon there is a parameter space in which the model is within the $2 \sigma$ observational contour.

From Fig. 8 we find that the models with larger values of $\alpha$ and $\beta$ tend to be favored due to the suppressed growth of matter perturbations in high redshifts. Case (iii) shown in Fig. 5 corresponds to such an example, in which case the model is inside the $90.0 \%$ CL observational contour. Case (i) in Fig. 5 is excluded at $99.0 \%$ CL because of large values of $f_{m} \sigma_{8}$ in both high and low redshifts. Case (ii) is outside the $95.4 \%$ CL contour due to the large values of $f_{m} \sigma_{8}$ in high redshifts in spite of the suppressed evolution of $f_{m} \sigma_{8}$ for $z \lesssim 0.5$. In Table we summarize the results of one-dimensional constraints for a given value of $\alpha$ (or a combination $\beta-\alpha / 2$ ).

Varying the dark energy density parameter in the range $0.70<\Omega_{\mathrm{DE}}^{(0)}<0.75$ does not change the above constraints significantly. Setting $\sigma_{8}(z=0)=0.75$, we find that the whole parameter space can be allowed at $1 \sigma$ CL. For lager $\sigma_{8}(z=0)$, observational constraints get tighter.

It should be noted again that the observational bounds in Fig. 8 correspond to the model parameter $s=0.2$. For the $s$ values close to 0 , the growth rate of matter perturbations gets smaller and approaches the $\Lambda$ CDM rate. Obviously such models should also be compatible with the RSD data. Note that the cross-correlation between the large-scale structure (LSS) and the integratedSachs-Wolfe (ISW) effect in CMB can also provide additional constraints on the extended Galileon model. The joint data analysis of the RSD and the LSS-ISW crosscorrelation is beyond the scope of our paper.

\section{CONCLUSIONS}

In this paper we have placed observational constraints on dark energy models based on $f(R)$ gravity, covariant Galileon, and extended Galileon from the latest data 


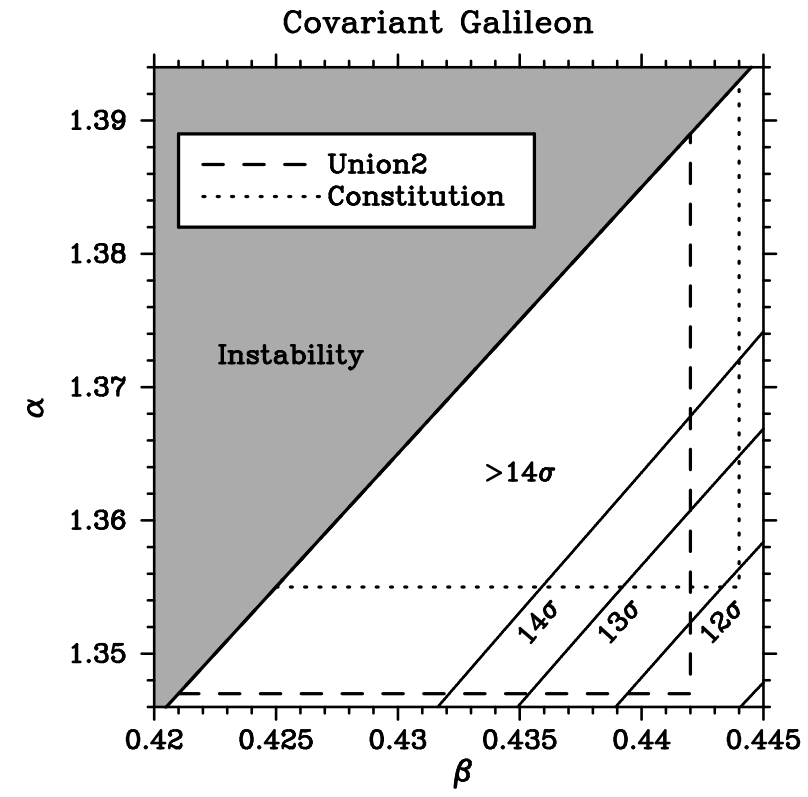

Figure 7: Constraints on the covariant Galileon model given by the functions (12)-(15) from the RSD data in the $(\alpha, \beta)$ plane for the scale $k^{-1}=30 h^{-1} \mathrm{Mpc}$ with $\sigma_{8}(z=0)=0.811$ and $\Omega_{\mathrm{DE}}^{(0)}=0.73$. The dashed and dotted lines represent the regions constrained by the background expansion history according to the Union2 and Constitution SN Ia data, respectively. In the grey region $(\alpha-2 \beta>0.505)$ there is an instability associated with negative values of $G_{\text {eff }}$ during the transition from the matter era to the accelerated epoch. The parameter space that is theoretically valid and consistent with background-level observations is excluded by the RSD data at more than $11 \sigma$ CL.

of galaxy redshift surveys (WiggleZ, SDSS LRG, BOSS, and 6dFGRS). In these models the General Relativistic behavior can be recovered at short distances under the chameleon mechanism or the Vainshtein mechanism. On scales relevant to large-scale structures the modification of gravity manifests itself for the growth rate of matter density perturbations. This growth rate is related to the peculiar velocities of galaxies, which can be constrained from the redshift-space distortions.

As an explicit example we studied the $f(R)$ model (6) proposed by $\mathrm{Hu}$ and Sawicki, which is general enough to cover basic properties of $f(R)$ dark energy models. At the level of perturbations this model mimics the $\Lambda \mathrm{CDM}$ for the redshift $z$ larger than the critical value $z_{c}$ given by Eq. (52), but the deviation appears for $z<z_{c}$. For smaller values of $n$ and $\lambda$ the transition redshift $z_{c}$ gets larger, so that the growth of matter perturbations is more significant at late times.

We placed observational bounds on the Hu-Sawicki $f(R)$ model in the $(n, \lambda)$ plane. Since the growth rate in this model depends on the scale of density perturbations, we derive constraints assuming three different wavenumbers of $k^{-1}=10,30$, and $60 h^{-1} \mathrm{Mpc}$ which are relevant

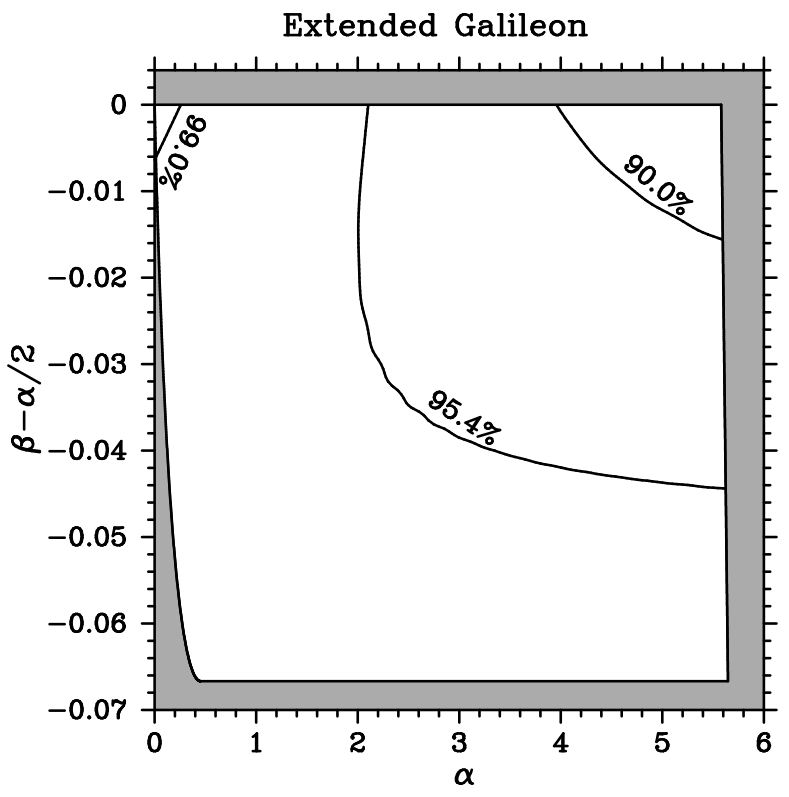

Figure 8: Constraints on the extended Galileon model given by the functions (25)-(28) from the RSD data in the $(\alpha, \beta)$ plane for the scale $k^{-1}=30 h^{-1} \mathrm{Mpc}$ with $\sigma_{8}(z=0)=$ 0.811 and $\Omega_{\mathrm{DE}}^{(0)}=0.72$. The grey scale region is theoretically excluded (see Fig. 4). The lower-left region separated by the $95.4 \%$ contour is excluded at more than $95.4 \%$ CL, and other contours have similar meanings.

to the galaxy redshift surveys considered here. As we see in Fig. [6 (which corresponds to $\sigma_{8}(z=0)=0.811$ and $\left.\Omega_{\mathrm{DE}}^{(0)}=0.73\right)$, the constraints on model parameters tend to be tighter for smaller scales. For $k^{-1}=60 h^{-1} \mathrm{Mpc}$ the models with $n<2$ and $\lambda<3$ are outside the $2 \sigma$ observational contour, whereas for $k^{-1}=10 h^{-1} \mathrm{Mpc}$ the models with $n<2$ and $\lambda<8$ are excluded at the $2 \sigma$ CL. These results show that the recent RSD data do not favor the large deviation of $f_{m} \sigma_{8}$ from that in the $\Lambda \mathrm{CDM}$, whose property can be confirmed in Figs. 1 and 2

For covariant Galileon there is a tracker solution along which the equation of state $w_{\mathrm{DE}}$ of dark energy changes from -2 (matter era) to -1 (de Sitter era). The likelihood analysis of SN Ia, CMB, and BAO shows that only the late-time tracking solution is allowed from the data. Using the parameter space constrained from the background cosmology, we found that the covariant Galileon model is excluded at more than $8 \sigma$ CL for $\sigma_{8}(z=0)$ ranging in the region $0.75<\sigma_{8}(z=0)<0.85$. This is associated with the fact that the cosmic growth rate for the covariant Galileon is much larger than that in the $\Lambda \mathrm{CDM}$, as confirmed in Fig. 3. In the range $10 h^{-1} \mathrm{Mpc}$ $\lesssim k^{-1} \lesssim 60 h^{-1} \mathrm{Mpc}$ the evolution of $f_{m} \sigma_{8}$ is practically independent of the scales.

In the extended Galileon scenario the equation of state for the tracker is given by $w_{\mathrm{DE}}=-1-s$ during the matter era, where $s$ is a positive constant. For the model 
with $s=0.2$ we found that there is a parameter space which is inside the $2 \sigma$ observational contour. As we see in Fig. 8, the models with larger values of $\alpha$ and $\beta$ tend to be favored from the RSD data.

According to the latest data release of Planck, the dark energy density parameter is constrained to be $\Omega_{\mathrm{DE}}^{(0)}=$ $0.686 \pm 0.020\left(68 \%\right.$ CL) 82. Varying $\Omega_{\mathrm{DE}}^{(0)}$ in this range, the allowed regions tend to be a little bit narrower in all three models. However, this does not give rise to any qualitatively different change to our results.

We have thus shown that the recent observations of redshift-space distortions are already quite powerful to constrain a number of modified gravity models. In upcoming future we should obtain more $f \sigma_{8}$ measurements that are more precise and/or at different redshifts by larger data of ongoing surveys or near-future projects such as Subaru/FMOS, Subaru/PFS, HETDEX and so on. We hope that we will be able to approach the origin of dark energy in the foreseeable future.

\section{ACKNOWLEDGEMENTS}

The authors were supported by the Grant-in-Aid for Scientific Research Fund of the JSPS (Grants No. 23684007 for TT and No. 24540286 for ST) and the Fund for Scientific Research on Innovative Areas (JSPS Grant No. 21111006 for ST).

\section{Note added.}

After the first submission of this paper, several related papers appeared [81, 83 85].
1. Barreira et al. 81] derived observational constraints on the covariant Galileon model, but without using the RSD constraints. They argued that the use of RSD constraints would induce significant uncertainties by the non-linear effects of the Vainshtein mechanism. Recently, Li et al. [83] showed that, in the DGP and Galileon models with the field self-interaction $X \square \phi$, the non-linear effects start to affect the velocity field even for $k \sim 0.05 \mathrm{~h} \mathrm{Mpc}^{-1}$. Wyman et al. 84. carried out the $N$-body simulations for a phenomenological model with a standard $\Lambda$ CDM expansion history and a Galileontype scalar field. Although these analyses do not cover the models with the full covariant and extended Galileon terms discussed in this paper, it will be certainly of interest to study how the non-linear effects can affect the constraints derived in our paper.

2. Neveu et al. 85] showed that, for the covariant Galileon, there is some allowed parameter space consistent with the RSD data. They pointed out that this difference may come from whether or not the AlcockPaczynski effect is taken into account or from the difference of normalization of $\sigma_{8}(z=0)$. We confirmed that, unlike our work, Neveu et al. did not impose the existence of a future de Sitter solution. In this case there is one free parameter $\left(c_{2}\right.$ or $\left.c_{3}\right)$ in addition to $\alpha$ and $\beta$. In fact, we checked that their best-fit model parameters do not belong to the theoretically allowed region constrained in Refs. 45, 46]. Our assumption for the existence of the de Sitter solution is reasonable, because the solutions with different initial conditions converge to the attractor of cosmic acceleration.
[1] A. G. Riess et al. [Supernova Search Team Collaboration], Astron. J. 116, 1009 (1998); S. Perlmutter et al. [Supernova Cosmology Project Collaboration], Astrophys. J. 517, 565 (1999).

[2] D. N. Spergel et al. [WMAP Collaboration], Astrophys. J. Suppl. 148, 175 (2003).

[3] M. Tegmark et al. [SDSS Collaboration], Phys. Rev. D 69, 103501 (2004).

[4] T. P. Sotiriou and V. Faraoni, Rev. Mod. Phys. 82, 451 (2010).

[5] E. J. Copeland, M. Sami and S. Tsujikawa, Int. J. Mod. Phys. D 15, 1753 (2006); S. Tsujikawa, Lect. Notes Phys. 800, 99 (2010).

[6] A. De Felice and S. Tsujikawa, Living Rev. Rel. 13, 3 (2010).

[7] T. Clifton, P. G. Ferreira, A. Padilla and C. Skordis, Phys. Rept. 513, 1 (2012).

[8] S. Capozziello, Int. J. Mod. Phys. D 11, 483 (2002); S. Capozziello, S. Carloni and A. Troisi, Recent Res. Dev. Astron. Astrophys. 1, 625 (2003); S. M. Carroll, V. Duvvuri, M. Trodden and M. S. Turner, Phys. Rev. D 70, 043528 (2004).

[9] L. Amendola, Phys. Rev. D 60, 043501 (1999);
J. P. Uzan, Phys. Rev. D 59, 123510 (1999); T. Chiba, Phys. Rev. D 60, 083508 (1999); N. Bartolo and M. Pietroni, Phys. Rev. D 61023518 (1999); F. Perrotta, C. Baccigalupi and S. Matarrese, Phys. Rev. D 61, 023507 (1999).

[10] G. R. Dvali, G. Gabadadze and M. Porrati, Phys. Lett. B 485, 208 (2000).

[11] A. Nicolis, R. Rattazzi and E. Trincherini, Phys. Rev. D 79, 064036 (2009).

[12] C. Deffayet, G. Esposito-Farese and A. Vikman, Phys. Rev. D 79, 084003 (2009); C. Deffayet, S. Deser and G. Esposito-Farese, Phys. Rev. D 80, 064015 (2009).

[13] S. M. Carroll, I. Sawicki, A. Silvestri and M. Trodden, New J. Phys. 8, 323 (2006); R. Bean, D. Bernat, L. Pogosian, A. Silvestri and M. Trodden, Phys. Rev. D 75, 064020 (2007); L. Pogosian and A. Silvestri, Phys. Rev. D 77, 023503 (2008); B. Li and J. D. Barrow, Phys. Rev. D 75, 084010 (2007).

[14] S. Tsujikawa, Phys. Rev. D 76, 023514 (2007).

[15] A. Lue, R. Scoccimarro and G. D. Starkman, Phys. Rev. D 69, 124015 (2004); K. Koyama and R. Maartens, JCAP 0601, 016 (2006).

[16] A. De Felice, R. Kase and S. Tsujikawa, Phys. Rev. D 
83, 043515 (2011).

[17] A. De Felice, T. Kobayashi and S. Tsujikawa, Phys. Lett. B 706, 123 (2011).

[18] G. W. Horndeski, Int. J. Theor. Phys. 10, 363 (1974).

[19] C. Deffayet, X. Gao, D. A. Steer and G. Zahariade, Phys. Rev. D 84, 064039 (2011); C. Charmousis, E. J. Copeland, A. Padilla and P. M. Saffin, Phys. Rev. Lett. 108, 051101 (2012).

[20] T. Kobayashi, M. Yamaguchi and J. Yokoyama, Prog. Theor. Phys. 126, 511 (2011).

[21] N. Kaiser, Mon. Not. Roy. Astron. Soc. 227, 1 (1987).

[22] M. Tegmark et al. [SDSS Collaboration], Astrophys. J. 606, 702 (2004).

[23] W. J. Percival et al. [The 2dFGRS Collaboration], Mon. Not. Roy. Astron. Soc. 353, 1201 (2004).

[24] M. Tegmark et al. [SDSS Collaboration], Phys. Rev. D 74, 123507 (2006).

[25] K. Yamamoto, T. Sato and G. Huetsi, Prog. Theor. Phys. 120, 609 (2008).

[26] L. Guzzo et al., Nature 451, 541 (2008).

[27] C. Blake et al., Mon. Not. Roy. Astron. Soc. 415, 2876 (2011).

[28] L. Samushia, W. J. Percival and A. Raccanelli, Mon. Not. Roy. Astron. Soc. 420, 2102 (2012).

[29] B. A. Reid, L. Samushia, M. White, W. J. Percival, M. Manera, N. Padmanabhan, A. J. Ross and A. G. Sanchez et al., Mon. Not. Roy. Astron. Soc. 426, 2719 (2012).

[30] F. Beutler, C. Blake, M. Colless, D. H. Jones, L. StaveleySmith, G. B. Poole, L. Campbell and Q. Parker et al., arXiv:1204.4725 [astro-ph.CO].

[31] L. Samushia, B. A. Reid, M. White, W. J. Percival, A. J. Cuesta, L. Lombriser, M. Manera and R. C. Nichol et al., Mon. Not. Roy. Astron. Soc. 429, 1514 (2013).

[32] M. J. Hudson and S. J. Turnbull, Astrophys. J. 751, L30 (2012).

[33] S. Tsujikawa, A. De Felice and J. Alcaniz, JCAP 1301, 030 (2013).

[34] J. Khoury and A. Weltman, Phys. Rev. Lett. 93, 171104 (2004); Phys. Rev. D 69, 044026 (2004).

[35] A. I. Vainshtein, Phys. Lett. B 39, 393 (1972).

[36] K. Hinterbichler and J. Khoury, Phys. Rev. Lett. 104, 231301 (2010); K. Hinterbichler, J. Khoury, A. Levy and A. Matas, Phys. Rev. D 84, 103521 (2011).

[37] P. Brax, C. van de Bruck, A. -C. Davis and D. Shaw, Phys. Rev. D 82, 063519 (2010).

[38] S. Tsujikawa, K. Uddin, S. Mizuno, R. Tavakol and J. Yokoyama, Phys. Rev. D 77, 103009 (2008); R. Gannouji, B. Moraes, D. F. Mota, D. Polarski, S. Tsujikawa and H. A. Winther, Phys. Rev. D 82, 124006 (2010).

[39] Y. -S. Song, W. Hu and I. Sawicki, Phys. Rev. D 75, 044004 (2007).

[40] Y. -S. Song, H. Peiris and W. Hu, Phys. Rev. D 76, 063517 (2007).

[41] L. Lombriser, A. Slosar, U. Seljak and W. Hu, Phys. Rev. D 85, 124038 (2012).

[42] K. Koyama, A. Taruya and T. Hiramatsu, Phys. Rev. D 79, 123512 (2009); G. B. Zhao et al., Phys. Rev. D 81, 103510 (2010); T. Narikawa and K. Yamamoto, Phys. Rev. D 81, 043528 (2010); K. Yamamoto, G. Nakamura, G. Hutsi, T. Narikawa and T. Sato, Phys. Rev. D 81, 103517 (2010).

[43] E. Jennings, C. M. Baugh, B. Li, G. -B. Zhao and K. Koyama, Mon. Not. Roy. Astron. Soc. 425, 2128
(2012); B. Li, W. A. Hellwing, K. Koyama, G. -B. Zhao, E. Jennings and C. M. Baugh, Mon. Not. Roy. Astron. Soc. 428, 743 (2013).

[44] R. Gannouji and M. Sami, Phys. Rev. D 82, 024011 (2010).

[45] A. De Felice and S. Tsujikawa, Phys. Rev. Lett. 105, 111301 (2010).

[46] A. De Felice and S. Tsujikawa, Phys. Rev. D 84, 124029 (2011).

[47] S. A. Appleby and E. V. Linder, JCAP 1208, 026 (2012).

[48] C. M. Will, Living Rev. Rel. 9, 3 (2005).

[49] A. A. Starobinsky, Phys. Lett. B 91, 99 (1980).

[50] J. A. R. Cembranos, Phys. Rev. D 73, 064029 (2006); I. Navarro and K. Van Acoleyen, JCAP 0702, 022 (2007); T. Faulkner, M. Tegmark, E. F. Bunn and Y. Mao, Phys. Rev. D 76, 063505 (2007).

[51] S. Capozziello and S. Tsujikawa, Phys. Rev. D 77, 107501 (2008).

[52] W. Hu and I. Sawicki, Phys. Rev. D 76, 064004 (2007).

[53] A. A. Starobinsky, JETP Lett. 86, 157 (2007).

[54] S. A. Appleby and R. A. Battye, Phys. Lett. B 654, 7 (2007).

[55] S. Tsujikawa, Phys. Rev. D 77, 023507 (2008).

[56] E. V. Linder, Phys. Rev. D 80, 123528 (2009).

[57] C. Deffayet, G. R. Dvali, G. Gabadadze and A. I. Vainshtein, Phys. Rev. D 65, 044026 (2002).

[58] M. Porrati, Phys. Lett. B 534, 209 (2002); M. A. Luty, M. Porrati and R. Rattazzi, JHEP 0309, 029 (2003).

[59] A. Nicolis and R. Rattazzi, JHEP 0406, 059 (2004); D. Gorbunov, K. Koyama and S. Sibiryakov, Phys. Rev. D 73, 044016 (2006).

[60] M. Fairbairn and A. Goobar, Phys. Lett. B 642, 432 (2006); R. Maartens and E. Majerotto, Phys. Rev. D 74, 023004 (2006); U. Alam and V. Sahni, Phys. Rev. D 73, 084024 (2006); Y. S. Song, I. Sawicki and W. Hu, Phys. Rev. D 75, 064003 (2007); J. Q. Xia, Phys. Rev. D 79, 103527 (2009).

[61] S. Nesseris, A. De Felice and S. Tsujikawa, Phys. Rev. D 82, 124054 (2010).

[62] R. Kimura and K. Yamamoto, JCAP 1104, 025 (2011).

[63] G. Dvali and M. S. Turner, arXiv:astro-ph/0301510.

[64] R. Kimura, T. Kobayashi and K. Yamamoto, Phys. Rev. D 85, 123503 (2012).

[65] A. De Felice and S. Tsujikawa, JCAP 1202, 007 (2012).

[66] A. De Felice and S. Tsujikawa, JCAP 1203, 025 (2012).

[67] T. V. Ruzmaikina and A. A. Ruzmaikin, Zh. Eksp. Teor. Fiz. 57, 680 (1969) [Sov. Phys. - JETP 30, 372 (1970)]; B. N. Breizman, V. Ts. Gurovich and V. P. Sokolov, Zh. Eksp. Teor. Fiz. 59, 288 (1970) [Sov. Phys. - JETP 32, 155 (1971)]; R. Kerner, Gen. Rel. Grav. 14, 453 (1982).

[68] V. Muller, H. J. Schmidt and A. A. Starobinsky, Phys. Lett. B 202, 198 (1988).

[69] V. Faraoni, Phys. Rev. D 72, 061501 (2005); V. Faraoni and S. Nadeau, Phys. Rev. D 72, 124005 (2005).

[70] L. Amendola, R. Gannouji, D. Polarski and S. Tsujikawa, Phys. Rev. D 75, 083504 (2007); L. Amendola and S. Tsujikawa, Phys. Lett. B 660, 125 (2008).

[71] B. Li and J. D. Barrow, Phys. Rev. D 75, 084010 (2007).

[72] J. M. Bardeen, Phys. Rev. D 22, 1882 (1980).

[73] A. A. Starobinsky, JETP Lett. 68, 757 (1998); B. Boisseau, G. Esposito-Farese, D. Polarski and A. A. Starobinsky, Phys. Rev. Lett. 85, 2236 (2000); A. De Felice, S. Mukohyama and S. Tsujikawa, Phys. Rev. D82, 023524 (2010). 
[74] E. Komatsu et al. [WMAP Collaboration], Astrophys. J. Suppl. 192, 18 (2011).

[75] D. Rapetti, C. Blake, S. W. Allen, A. Mantz, D. Parkinson and F. Beutler, arXiv:1205.4679 [astro-ph.CO].

[76] S. Tsujikawa, R. Gannouji, B. Moraes and D. Polarski, Phys. Rev. D 80, 084044 (2009).

[77] S. Tsujikawa, K. Uddin and R. Tavakol, Phys. Rev. D 77, 043007 (2008).

[78] H. Motohashi, A. A. Starobinsky and J. Yokoyama, Int. J. Mod. Phys. D 18, 1731 (2009).

[79] S. Appleby and E. V. Linder, JCAP 1203, 043 (2012).

[80] A. Barreira, B. Li, C. M. Baugh and S. Pascoli, Phys. Rev. D 86, 124016 (2012).
[81] A. Barreira, B. Li, A. Sanchez, C. M. Baugh and S. Pascoli, arXiv:1302.6241 [astro-ph.CO].

[82] P. A. R. Ade et al. [Planck Collaboration], arXiv:1303.5076 [astro-ph.CO].

[83] B. Li, G. -B. Zhao and K. Koyama, arXiv:1303.0008 [astro-ph.CO].

[84] M. Wyman, E. Jennings and M. Lima, arXiv:1303.6630 [astro-ph.CO].

[85] J. Neveu, V. Ruhlmann-Kleinder, A. Conley, N. Palanque-Delabrouille, P. Asitier, J. Guy and E. Babichev, arXiv:1302.2786 [gr-qc]. 